\title{
Guidelines
}

\section{S2K Guideline for Diagnosis of Idiopathic Pulmonary Fibrosis}

\author{
Jürgen Behr ${ }^{\mathrm{a}}$ Andreas Günther ${ }^{\mathrm{b}}$ Francesco Bonella ${ }^{c}$ Julien Dinkel ${ }^{\mathrm{d}}$ Ludger Fink $^{\mathrm{e}}$ \\ Thomas Geiser $^{f}$ Klaus Geissler ${ }^{g}$ Sven Gläser ${ }^{h}$ Sabin Handzhiev ${ }^{i}$ Danny Jonigk ${ }^{j} \quad$ Dirk Koschel $^{k}$ \\ Michael Kreuter ${ }^{\prime}$ Gabriela Leuschner ${ }^{a}$ Philipp Markart ${ }^{m}$ Antje Prasse ${ }^{n}$ Nicolas Schönfeld ${ }^{\circ}$ \\ Jonas Christian Schupp ${ }^{p}$ Helmut Sitter ${ }^{q} \quad$ Joachim Müller-Quernheim ${ }^{r} \quad$ Ulrich Costabel $^{c}$
}

aDepartment of Internal Medicine V, Ludwig-Maximilians-University (LMU) of Munich, Comprehensive Pneumology Center, Member of the German Center for Lung Research (Deutsches Zentrum für Lungenforschung, DZL), Munich, Germany; bection of Fibrotic Lung Diseases, University Hospital Giessen and Marburg, Giessen Campus, Justus Liebig University Giessen, Agaplesion Pneumological Clinic Waldhof-Elgershausen, University of Giessen Marburg Lung Center, Member of the German Center for Lung Research (Deutsches Zentrum für Lungenforschung, DZL), Giessen, Germany; 'Center for Interstitial and Rare Lung Diseases, Pneumology Department, Ruhrlandklinik - University Hospital, University Duisburg-Essen, Essen, Germany; 'Department of Radiology, University Hospital, LMU, and Asklepios Specialty Hospitals Munich Gauting, Member of the German Center for Lung Research (Deutsches Zentrum für Lungenforschung, DZL), Munich, Germany; eInstitute of Pathology and Cytology, Supraregional Joint Practice for Pathology (Überregionale Gemeinschaftspraxis für Pathologie, ÜGP), Member of the German Center for Lung Research (Deutsches Zentrum für

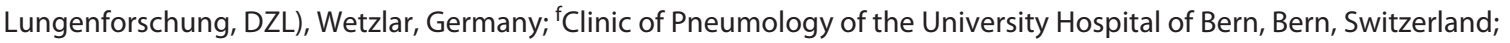

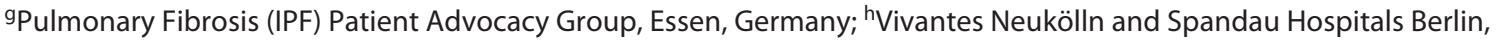
Department of Internal Medicine - Pneumology and Infectiology as well as Greifswald Medical School, University of Greifswald, Greifswald, Germany; 'Clinical Department of Pneumology, University Hospital Krems, Krems, Austria; jInstitute of Pathology, Hanover Medical School, Member of the German Center for Lung Research (Deutsches Zentrum

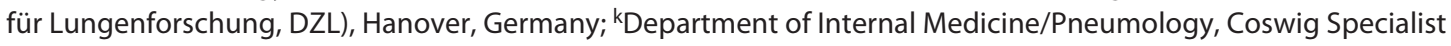
Hospital, Center for Pneumology, Allergology, Respiratory Medicine, Thoracic Surgery and Medical Clinic 1, Pneumology Department, Carl Gustav Carus University Hospital, Dresden, Germany; 'Center for Interstitial and Rare Lung Diseases, Pneumology and Respiratory Medicine, Thorax Clinic, University Hospital Heidelberg, Member of German Center for Lung Research (Deutsches Zentrum für Lungenforschung, DZL), Heidelberg, Germany; ${ }^{\mathrm{m}}$ Section of Fibrotic Lung Diseases, University Hospital Giessen and Marburg, Giessen Campus, Justus Liebig University Giessen, University of Giessen Marburg Lung Center, as well as the Fulda Campus of the Medical University of Marburg, Med. Clinic V, Member of German Center for Lung Research (Deutsches Zentrum für Lungenforschung, DZL), Giessen, Germany; 'Department of Pneumology, Hanover Medical School and Clinical Research Center Fraunhofer Institute ITEM, Member of the German Center for Lung Research (Deutsches Zentrum für Lungenforschung, DZL), Hanover, Germany; ${ }^{\circ}$ Pneumology Clinic, Part of the Heckeshorn

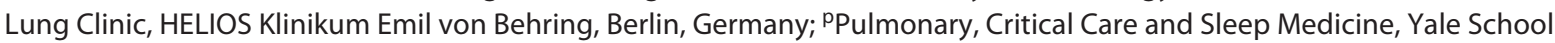
of Medicine, Yale University, New Haven, CT, USA; IInstitute for Surgical Research, Philipps-University Marburg, Marburg, Germany; 'Department of Pneumology, Medical Center - University of Freiburg, Faculty of Medicine - University of Freiburg, Freiburg, Germany

\section{Keywords}

Idiopathic pulmonary fibrosis - Diagnosis - Guideline · Biopsy · High-resolution computed tomography . Bronchoalveolar lavage · Serology

\begin{abstract}
Idiopathic pulmonary fibrosis (IPF) is a severe and often fatal disease. Diagnosis of IPF requires considerable expertise and experience. Since the publication of the international IPF guideline in the year 2011 and the update 2018 several stud-
\end{abstract}

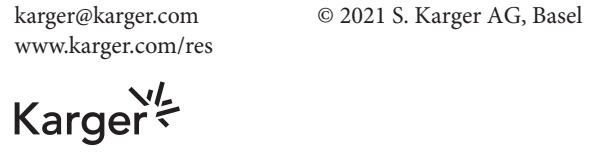

\footnotetext{
Jürgen Behr

Medizinische Klinik und Poliklinik V, LMU Klinikum

Campus Großhadern und Innenstadt

Marchioninistr. 15, DE-81377 Munich (Germany)

juergen.behr@med.uni-muenchen.de
} 
ies and technical advances have occurred, which made a new assessment of the diagnostic process mandatory. The goal of this guideline is to foster early, confident, and effective diagnosis of IPF. The guideline focusses on the typical clinical context of an IPF patient and provides tools to exclude known causes of interstitial lung disease including standardized questionnaires, serologic testing, and cellular analysis of bronchoalveolar lavage. High-resolution comput- ed tomography remains crucial in the diagnostic workup. If it is necessary to obtain specimens for histology, transbronchial lung cryobiopsy is the primary approach, while surgical lung biopsy is reserved for patients who are fit for it and in whom a bronchoscopic diagnosis did not provide the information needed. After all, IPF is a diagnosis of exclusion and multidisciplinary discussion remains the golden standard of diagnosis.

\section{Table of Contents}

\section{About This Guideline}

1.1. Responsible Professional Association

1.2. Important Note

\section{Scope of Application and Purpose of the Guideline}

2.1. Rationale for Selecting the Guideline Topic

2.2. Objective of the Guideline

2.3. Target Patient Group

2.4. Coverage Area

2.5. Target User Groups/Addressees

3. Composition of the Guideline Panel

3.1. Coordination and Editing

3.2. Professional Associations and Organizations Involved

3.3. Methodological Support

\section{Accuracy of Methods}

4.1. Drafting of the Guideline/Consenting

4.2. Statements

4.3. Expert Consensus (EC)

4.4. External Review and Approval

\section{Editorial Independence}

\section{Preparation and Implementation}

\section{Validity and Updating Process}




\title{
8. Clinical Presentation
}

\author{
8.1. Epidemiology \\ 8.2. Characteristic Features \\ 8.3. Risk Factors \\ 8.4. Comorbidities \\ 8.5. Progression and Follow-Up Monitoring \\ 8.6. Prognosis \\ 8.7. Diagnosis
}

\section{Imaging}

9.1. Usual Interstitial Pneumonia Pattern on HRCT

9.2. HRCT Patterns

9.3. UIP Pattern

9.4. Probable UIP Pattern

9.5. Indeterminate for UIP

9.6. Alternative Pattern

9.7. CT Findings in Acute Exacerbation

\section{Histopathology Aspects}

10.1. Diagnostic Procedure

\section{Clinically Relevant Questions}

Question 1: should patients with newly detected ILD of unknown cause who are clinically suspected of having IPF undergo a detailed, prompted history of (previous) medication use and inhalational exposures at home and at work, to exclude potential causes of the ILD?

Question 2: should patients with newly detected ILD of unknown cause who are clinically suspected of having IPF undergo serological testing to exclude or diagnose an underlying autoimmune disorder?

Question 3: should patients with newly detected ILD of unknown cause who are clinically suspected of having IPF undergo cellular analysis of their BAL fluid?

Question 4: for patients with newly detected ILD of unknown cause who are clinically suspected of having IPF, should surgical lung biopsy (SLB) be performed to ascertain the histopathology diagnosis of UIP pattern?

Question 5: should a transbronchial lung biopsy (forceps) be performed for patients who are suspected of having IPF to ascertain the histopathology diagnosis of UIP pattern?

Question 6: for patients with newly detected ILD of unknown cause who are clinically suspected of having IPF, is transbronchial lung cryobiopsy (TBLC) a reasonable alternative to SLB to sufficiently ascertain the histopathology diagnosis of UIP pattern? 
Question 7: should patients with newly detected ILD of unknown cause who are clinically suspected of having IPF be diagnosed in the context of an MDD?

Question 8: should patients with newly detected ILD of unknown cause who are clinically suspected of having IPF undergo serum biomarker measurement to corroborate the IPF diagnosis?

\section{Diagnostic Criteria}

\section{Scientific Questions and Outlook}

\section{Summary of Recommendations}

\section{Acknowledgements}

\section{Conflict of Interest Statement}

\section{References}




\section{About This Guideline}

\subsection{Responsible Professional Association}

German Respiratory Society (Deutsche Gesellschaft

für Pneumologie und Beatmungsmedizin, DGP)

Robert-Koch-Platz 9

10115 Berlin.

\subsection{Important Note}

Medical science is continuously advancing. The information provided in this guideline - in particular concerning diagnostic and treatment procedures - can therefore only reflect the state of knowledge at the time of going to press. The application of therapy, medication, and dosage recommendations remains the responsibility of the user.

This work and the component parts thereof are copyrighted. The use of this work other than as permitted by copyright law is prohibited and liable to prosecution and shall require the editorial office's written consent. No part of this publication may be reproduced in any form without the express written consent of the authors. This includes, in particular, reproductions, translations, microfilming, as well as the entry, use, and exploitation of this work in electronic systems, intranets, and the Internet.

\section{Scope of Application and Purpose of the Guideline}

\subsection{Rationale for Selecting the Guideline Topic}

This guideline for the diagnosis of idiopathic pulmonary fibrosis (IPF) is an update to the [German] S2K guideline for diagnosis and therapy of IPF published in 2013 in PNEUMOLOGIE [1]. Since the publication of the guideline in 2013, an update on therapies was published in 2017 [2]. A relevant number of studies have been published in the meantime - particularly with respect to the diagnosis of IPF - which need to be considered. The body of scientific evidence has grown considerably, and this update only concerns the field of diagnosis and is published as a supplement to the original guideline.

\subsection{Objective of the Guideline}

The main objective of this guideline is to improve and standardize the diagnosis of IPF. It aims to achieve the highest possible diagnostic confidence with the lowest possible invasiveness of the diagnostic methods used. Another objective is to diagnose patients at an early stage. Improvements in the quality of care are intended to be achieved by the following:

- identifying affected patients at an early stage;

- standardizing diagnostic procedures;
- improving the interpretation of findings;

- streamlining the diagnostic strategy and prioritizing noninvasive investigational tests.

\subsection{Target Patient Group}

The guideline focuses on the group of patients suffering from IPF. However, it is also relevant for patients with any interstitial lung disease (ILD) as it is used to distinguish IPF from other types of ILD.

\subsection{Coverage Area}

The area covered by the guideline includes outpatient as well as inpatient structures responsible for diagnosing patients. The guideline is relevant for both general practitioners and specialists.

\subsection{Target User Groups/Addressees}

This guideline is intended for medical and nonmedical professionals (physicians and nonphysicians), who may be involved in the treatment of IPF in one way or the other. They include pneumologists, internal medicine specialists, cardiologists, radiologists, pathologists, thoracic surgeons, basic scientists, nurses, patients, patient advocacy groups, and interested laypersons). The guideline serves as a source of information for medical assistant professionals.

\section{Composition of the Guideline Panel}

\subsection{Coordination and Editing}

Prof. Dr. Med. Jürgen Behr, Coordinator and Editor

Department of Internal Medicine V, LMU University

Hospital Munich and Asklepios Specialty Hospitals Munich-Gauting.

\subsection{Professional Associations and Organizations Involved} German Respiratory Society (Deutsche Gesellschaft für Pneumologie und Beatmungsmedizin e.V., DGP)

Robert-Koch-Platz 9

10115 Berlin.

Delegated representatives: J. Behr, A. Günther, F. Bonella, D. Koschel, M. Kreuter, G. Leuschner, P. Markart, S. Gläser, A. Prasse, N. Schönfeld, J. Schupp, J. Müller-Quernheim, and U. Costabel

German Society of Radiology (Deutsche Röntgengesellschaft e.V.)

Ernst-Reuter-Platz 10

10587 Berlin.

Delegated representative: J. Dinkel 
German Society of Pathology (Deutsche Gesellschaft für Pathologie)

Robert-Koch-Platz 9

10115 Berlin.

Delegated representatives: D. Jonnigk, L. Fink

Federal Association of German Pathologists (Bundesverband Deutscher Pathologen e. V.)

Berlin, www.pathologie.de.

Delegated representatives: D. Jonnigk, L. Fink

Austrian Society for Pneumology (Österreichische Gesellschaft für Pneumologie)

C/o Mondial Congress \& Events

Operngasse 20B

1040 Vienna.

Delegated representative: S. Handzhiev

Swiss Society for Pneumology (Schweizerische Gesellschaft für Pneumologie)

Secretariat

Reinacherstrasse 131

CH-4053 Basel.

Delegated representative: T. Geiser

Patient Association for Pulmonary Fibrosis (Lungenfibrose e.V.)

Kupferdreher Str. 114

45257 Essen.

Delegated representative: K. Geißler

Association of the Scientific Medical Societies in Germany (Arbeitsgemeinschaft der Wissenschaftlichen Medizinischen Fachgesellschaften e.V., AWMF)

Birkenstr. 67

10559 Berlin.

Delegated representative: H. Sitter.

\subsection{Methodological Support}

The methods for the development of this guideline were facilitated by AWMF (Association of the Scientific Medical Societies in Germany).

\section{Accuracy of Methods}

The methods used to develop the guideline are described in the guideline report. The guideline panel decided to lean on and adapt the 2018 ATS-ERS-JRS-ALAT clinical practice guideline which used the GRADE meth- od characterized by the following: (a) systematic search with inclusion and exclusion criteria; (b) critical evaluation of the literature according to GRADE; (c) indication of recommendation levels and strength of evidence; (d) recognizable linking of recommendations and relevant literature; and (e) preparation of evidence tables for relevant studies [3].

The guideline and the associated document, including searches and evidence tables, are available as pdf files on the AWMF website (https://www.awmf.org/leitlinien/ detail/1l/020-016.html). Complementary to the international guideline, publications published after September 2017 (and thus not available for the international guideline) were considered.

This guideline was supported throughout its development by AWMF (Association of the Scientific Medical Societies in Germany) (H.M.). Voting in the consensus meetings took place in line with the nominal group technique process and was facilitated by H.M. The consensus process for this S2K guideline included the following elements: logical analysis (clinical algorithm), formal consensus building, evidence-basing, and decision analysis. The rules for S2K guidelines state that a solution has to be based on a clearly defined set of questions and derived in several steps using conditional logic (if/then logic). Clinical trials and meta-analyses are included as evidence basis. The procedure is to be presented in a simple, clear, and concise manner using graphic algorithms.

\subsection{Drafting of the Guideline/Consenting}

The first version of the guideline was drafted under the direction of lead author Jürgen Behr by the authors of the individual chapters. This version was then circulated by e-mail among all members of the guideline panel, modified, and eventually provided the basis for the consensus meeting held in Munich on August 9, 2019.

\subsection{Statements}

Statements are presentations or explanations of specific facts or questions without a direct call for action. They are adopted in the context of a formal consensus process, in line with the approach taken for recommendations, and may be based either on study results or expert opinion.

\subsection{Expert Consensus (EC)}

In the context of the S2K guideline, recommendations are based on expert consensus; a comprehensive and systematic review of the entire available literature was not carried out. Expert consensus was graded not through 
Table 1. Grading of recommendations

\begin{tabular}{lll}
\hline $\begin{array}{l}\text { Level of } \\
\text { recommendation }\end{array}$ & Description & Wording \\
\hline A & Strong recommendation & Shall \\
B & Weak recommendation & Should \\
0 & Conditional recommendation & Can \\
\hline
\end{tabular}

symbols or letters; rather, the strength of consensus is indicated by the wording used (shall/should/can), as shown in Table 1.

\subsection{External Review and Approval}

As part of the adoption process, the guideline is reviewed and consented by all medical societies involved.

\section{Editorial Independence}

The development of the guideline was funded by the German Respiratory Society (DGP) and the medical societies involved. Guideline development was editorially independent from the funding parties; there were no additional sponsors.

Funding was used exclusively to cover the cost of staff, office supplies, the procurement of literature, and for the consensus meeting (rent, technology, catering, facilitator fees, travel expenses, and accommodation costs).

Potential conflicts of interest were documented for all guideline panel members using standardized AWMF forms. The completed forms were reviewed and evaluated by the coordinators. The relationships and facts disclosed therein as well as the statemnets of conflicts of interest of the guideline panel members are presented in the guideline report published elsewhere (https://www.awmf.org/ leitlinien/detail/ll/020-016.html). Where a potential conflict of interest was identified, the respective members were interviewed. In conclusion, there were no conflicts of interest relevant enough to justify the exclusion of a member on the grounds of bias.

The issue of conflicts of interest was jointly discussed at the beginning of the consensus meeting for the drafting process. Further potential risks of bias were reduced by the formal consensus building process, the interdisciplinary and multi-professional drafting process, and the possibility of public review. We would like to thank the representatives and experts for serving exclusively on a pro bono basis.

\section{Preparation and Implementation}

The guideline is published on the websites of the AWMF and the German Respiratory Society as well as in the medical journal "Pneumologie." It will also be presented at professional conferences of the participating professional societies and, thus, be brought to the attention of those involved in patient care as well as interested parties.

\section{Validity and Updating Process}

The S2K guideline will be valid until updated. The next update is scheduled to be made after 5 years, that is, in 2024. In the event of an urgent need for modification, a new version may be drafted earlier. Comments and suggestions for the updating process are explicitly encouraged, and can be addressed to the guideline secretariat:

Univ.-Prof. Dr. Med. Jürgen Behr (project leader)

Department of Internal Medicine V

Comprehensive Pneumology Center Munich

Ludwig-Maximilians-Universität (LMU) München

Marchioninistr. 15, 81377 München

Juergen.behr@med.uni-muenchen.de.

\section{Clinical Presentation}

IPF is a chronic, progressive, and irreversible ILD [4, 5]. Intensive research in recent years has led to numerous insights into the underlying pathophysiological processes. These include, without being limited to, faulty repair mechanisms in epithelial cell dysfunction, fibroblast activation, oxidative stress, vascular remodeling, genetic modifications, and aging processes (senescence). The exact pathogenesis remains unknown [6].

\subsection{Epidemiology}

Epidemiological data are inhomogeneous due to different statistical collection methods and disease definitions. The reported incidence rates in Europe and North America are between 2.8 and 19 cases per 100,000 people per year [7]. IPF is the most common form of idiopathic interstitial pneumonia. With a prevalence of 8.2 cases per 100,000 , it is, however, a rare disease (orphan disease) [8]. Prevalence increases with age [7]. Familial clustering of IPF was observed in up to $11 \%$ of cases $[9,10]$. 
Evaluations of randomized studies [11-13], as well as data from national and international patient registries [9, 14-18], show that the onset of the disease is usually in the sixth decade of life, with an age peak between 60 and 70 years. Men are more frequently affected than women, and the majority of patients have a history of smoking. This clinical constellation should lead to the suspicion of IPF and be considered in the differential diagnosis as an important indicator for the further diagnostic approach.

Earlier onset is possible in familial IPF and has been described repeatedly $[17,19]$. The possibility of a pulmonary manifestation of an autoimmune disease should always be considered [20] if affected patients are younger.

\subsection{Characteristic Features}

The most common symptoms are shortness of breath (up to $85 \%$ of cases), initially on exertion, later also at rest, cough (up to $75 \%$ ), tiredness, and loss of appetite $[9,14$, $17,21]$. Symptoms usually are of insidious onset and increase over time. In exceptional cases, IPF may first present as an acute exacerbation, that is, acute worsening of dyspnea over just a few weeks.

Clinical examination reveals bibasilar inspiratory crackles (synonymous: velcro rales), a typical finding on auscultation that can be observed in $80-95 \%$ of patients $[9,14,17]$. Clubbing can be seen in $20-30 \%$ of patients [ 9 , $14,17]$. The lung function parameters of forced vital capacity (FVC) and total lung capacity (TLC), as well as diffusion capacity (TLCO) of the lungs, may be within the reference range at the time of diagnosis or already be reduced and will typically deteriorate further as the disease progresses $[9,11,13-16]$.

\subsection{Risk Factors}

Tobacco smoke undeniably increases the risk of developing IPF. Sixty to seventy percent of affected patients have a history of smoking $[9,12,14,15,22]$. IPF usually manifests years or decades after active smoking has been discontinued. According to data from the German INSIGHTS IPF registry, the average time between quitting smoking and the manifestation of IPF is 21 years [14]. At the time of diagnosis, only $1-6.8 \%$ of patients are still active smokers [9-11, 14-16].

Environmental factors such as exposure to asbestos, metal and wood dusts, chemicals, or contact with allergens usually found in hypersensitivity pneumonitis (HP; synonym: extrinsic allergic alveolitis), were found in up to $38 \%$ of IPF patients. However, a definite causal relationship could not be demonstrated $[9,14]$.
Genetic polymorphisms in the mucin $5 \mathrm{~B}$ gene (MUC5B) promoter, for example, variant rs35705950, or mutations in the telomerase encoding genes, for example, TERT or TERC, which cause telomere shortening, or in the surfactant proteins are thought to be associated with the development of IPF [23]. However, genetic testing is not routinely done at present.

\subsection{Comorbidities}

Comorbidities are generally more prevalent in patients with IPF than in the general population and are relevant for prognosis [24, 25]. Relevant comorbidities are cardiovascular and thromboembolic diseases, gastroesophageal reflux, lung cancer, depression, sleep-related respiratory disorders, and diabetes $[9,26,27]$. IPF can be accompanied by COPD or pulmonary emphysema in one-third of cases, as these diseases result from years of smoking $[9,26,27]$.

Lung cancer is diagnosed more frequently in IPF patients than in the general population, in 3\% of patients within the first year of diagnosis of IPF. The cumulative incidence reaches $11 \%$ in a 4 -year follow-up period and $54 \%$ in a 10 -year follow-up period $[27,28]$. A common risk factor for both diseases is not only smoking, but also similar pathophysiological mechanisms such as activation of the tyrosine kinase epidermal growth factor receptor, cellular aging processes, and genetic changes [23]. The presence of IPF limits the diagnostic and therapeutic options and goes along with a poorer prognosis of lung cancer and vice versa. This holds also true for the early stages of lung cancer $[23,29]$.

\subsection{Progression and Follow-Up Monitoring}

IPF progression is heterogeneous. Slow disease progression over several years and exacerbations are seen, as are rapidly progressing manifestations of the disease [21]. Follow-up assessments are usually performed at 3-4-month intervals.

Important parameters for assessing the course of the disease are lung function (FVC and TLC), blood gas analysis at rest and during exercise, TLCO, exercise tolerance (6-min walking test), quality of life (including the SGRQ or the K-BILD questionnaire) as well as imaging techniques (high-resolution computed tomography [HRCT]). In addition, cumulative scores such as the GAP and TORVAN index are used to assess disease severity and the associated prognosis [19]. Without treatment, FVC deteriorates by $200-280 \mathrm{~mL}$ per year $[22,26]$. The deterioration is not a linear process; that is, past FVC deterioration does not predict the future FVC trend [16]. 
Managing patients with this highly complex clinical condition in specialized ILD centers allows the comprehensive diagnostic evaluation and case-by-case decisionmaking in the context of institutional multidisciplinary ILD boards at the time of initial diagnosis. Periodic follow-up visits at the centers will ensure that patients receive the best possible treatment (e.g., participation in clinical studies). A common approach is that patients are alternately seen by a pulmonologist and by the ILD center.

\subsection{Prognosis}

The cause of death in IPF patients is more frequently the underlying pulmonary fibrosis than any comorbidity. In large pivotal studies, $5.7-8.3 \%$ of patients in the placebo groups died within a year versus only $3.5-6.7 \%$ of patients in the verum groups $[11,13]$.

Frequent hospitalizations negatively affect the prognosis of the disease. Acute exacerbation of IPF is associated with an in-hospital mortality $>50 \%$ and a median survival time of 3-4 months [30, 31].

\subsection{Diagnosis}

The diagnosis of IPF continues to be a diagnosis of exclusion. Given the different therapeutic approaches, the differentiation from other forms of chronic progressive pulmonary fibrosis is essential.

The diagnostic process requires detailed knowledge of differential diagnosis and a structured approach to reliably exclude known causes of ILDs [32].The differential diagnosis includes autoimmune diseases with pulmonary involvement, especially ILDs associated with rheumatoid arthritis or other types of connective tissue disease (CTD), drug-induced pneumonitis, pneumoconioses, chronic HP, chronic sarcoidosis, and, rarely, infections (e.g., tuberculosis). The average time from the onset of symptoms to the correct diagnosis is 1.5 years $[13,22]$.

\section{Imaging}

HRCT plays a central role in the diagnosis of IPF. A chest $\mathrm{x}$-ray alone is not suitable for identifying and further characterizing ILD due to the subtle and complex parenchymal changes.

The optimal technique for evaluating ILD is outlined below:

- HRCT technique:

- Spiral data, i.e., volumetric acquisition with smallest possible collimation, has largely replaced sequential
$\mathrm{CT}$ acquisition. It ensures an accurate analysis of threedimensional distribution patterns using multi-planar reformation and post-processing techniques such as MIP (maximum intensity projection) $[33,34]$. In addition, the volumetric acquisition is more suitable for follow-up monitoring.

- The fastest gantry rotation and pitch (ratio of table feed to $360^{\circ} \mathrm{CT}$ gantry rotation) should be used to reduce breathing and heart rate-related artifacts [35].

- The DRG Working Group on Diagnostic Radiology of Occupational and Environmental Diseases (Arbeitsgemeinschaft Diagnostische Radiologie arbeits- und umweltbedingter Erkrankungen, AG DrauE) has delivered recommendations for the Low-Dose-Volume HRCT of the lungs to ensure standardization of CT examinations when using different scanners [36].

- Number of CT images:

- The initial image is acquired under deep inspiration (volumetric acquisition) with the patient in supine position.

- A second image should be acquired of the entire chest during exhalation (sequential CT acquisition), if a pathology within the small airways is to be confirmed/ rejected (e.g., bronchiolitis, HP) [37].

- Optionally, a third image in prone position (sequential CT acquisition only in the lower field, as necessary) can be acquired to rule out posture-induced changes of lung parenchyma (hypostasis) [38].

- Breathing instructions must be given before each acquisition (preferably by the MTA rather than by automated voice messages) [39].

- CT scan in case of acute respiratory deterioration in patients with known ILD.

- Acute pulmonary embolism should always be ruled out in patients presenting with acute respiratory deterioration. A chest CT angiography should, therefore, be obtained, either as a stand-alone procedure or in addition to a non-contrast HRCT protocol, if respective symptoms are present.

- Acquisitions with the patient in supine position only are adequate to answer this question.

- Acute IPF exacerbation should be excluded.

\subsection{Usual Interstitial Pneumonia Pattern on HRCT}

Usual interstitial pneumonia (UIP) is characterized by distinctive HRCT findings, including honeycombing, traction bronchiectasis, and/or traction bronchiolectasis, fine reticulation often in conjunction with ground-glass opacity (GGO) of the lung parenchyma [40, 41]. 

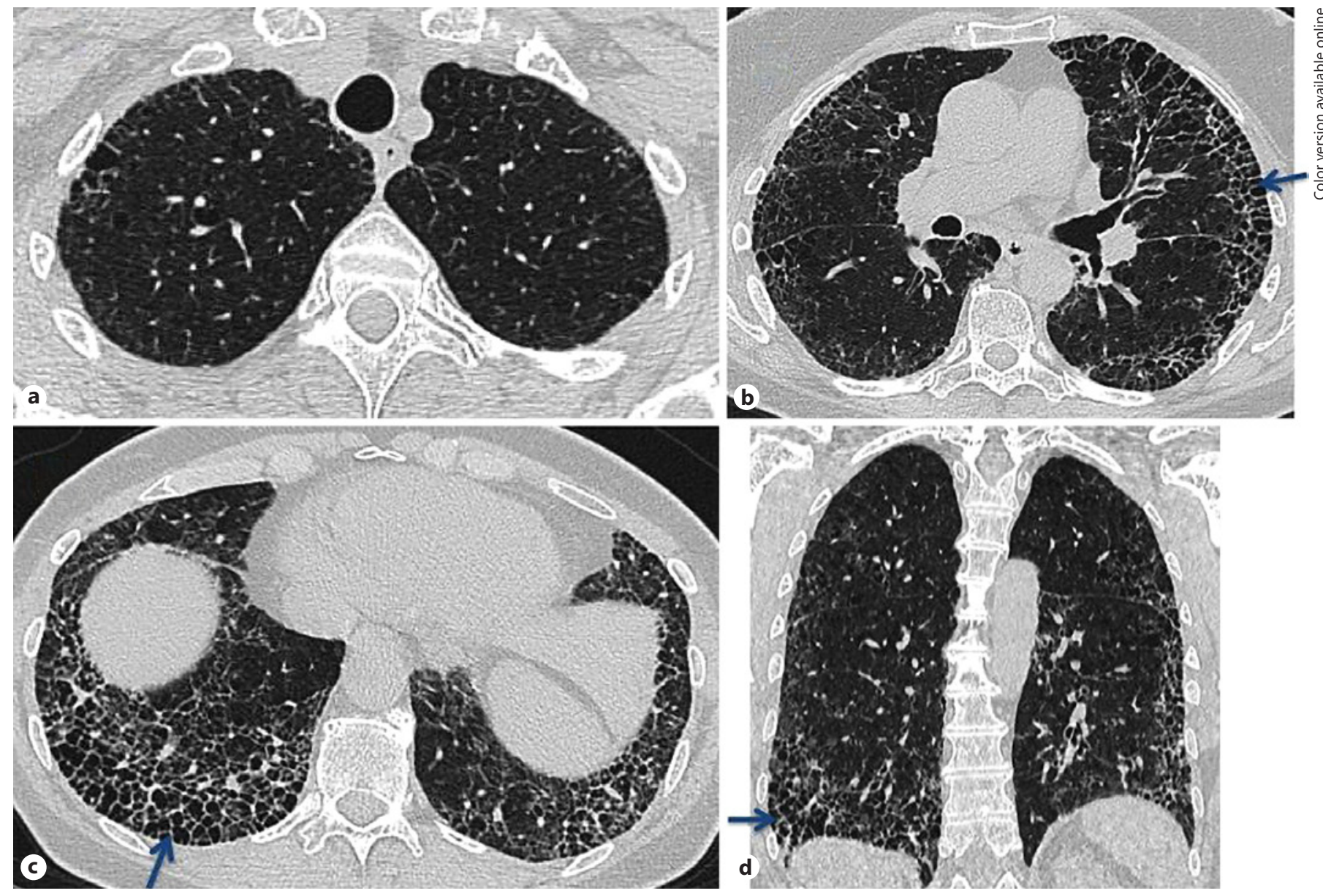

Fig. 1. UIP pattern on HRCT: axial (a-c) and coronal (d) CT image reconstructions of a patient with UIP pattern. Subpleural reticular abnormalities with traction bronchiectasis and honeycombing (arrows), and a distinct craniocaudal gradient in coronal reconstruction $(\mathbf{d})$.

\section{- Honeycombing:}

Honeycombing is characterized by clustered, cystic airspaces of typically consistent diameter $(3-10 \mathrm{~mm}$, but occasionally larger). They are usually located in the subpleural parenchyma and have well-defined walls. Honeycombing is commonly accompanied by a reticular pattern containing traction bronchiectasis and bronchiolectasis. It often presents as multiple layers of subpleural cysts on top of each other but may also present as a single layer. In these cases, distinguishing between honeycombing and paraseptal emphysema or traction bronchiolectasis may be difficult. Interobserver agreement regarding the identification of honeycombing is moderate, with disagreement most commonly due to another, similar subpleural pathology (e.g., traction bronchiolectasis, paraseptal emphysema, and subpleural cysts) $[42,43]$.
- Traction bronchiectasis and bronchiolectasis:

Traction bronchiectasis and bronchiolectasis are characterized by irregular dilatations of the lumen of bronchi/ bronchioles. It is usually peripheral/subpleural in UIP, often coexisting with honeycomb-like cysts, and may be best seen as peripheral traction bronchiolectasis. Traction bronchiectasis is considered a reliable sign of pulmonary fibrosis $[44,45]$. It can present as pronounced airway irregularity and is also called varicose bronchiectasis.

\section{- GGO:}

GGO is a common feature in UIP, but usually less widespread than the reticular patterns. GGO is an increase in density of the low-density lung parenchyma, whereby vascular and bronchial walls can be identified within the densification. It is important to distinguish between "pure" GGO and GGO superimposed on fine re- 


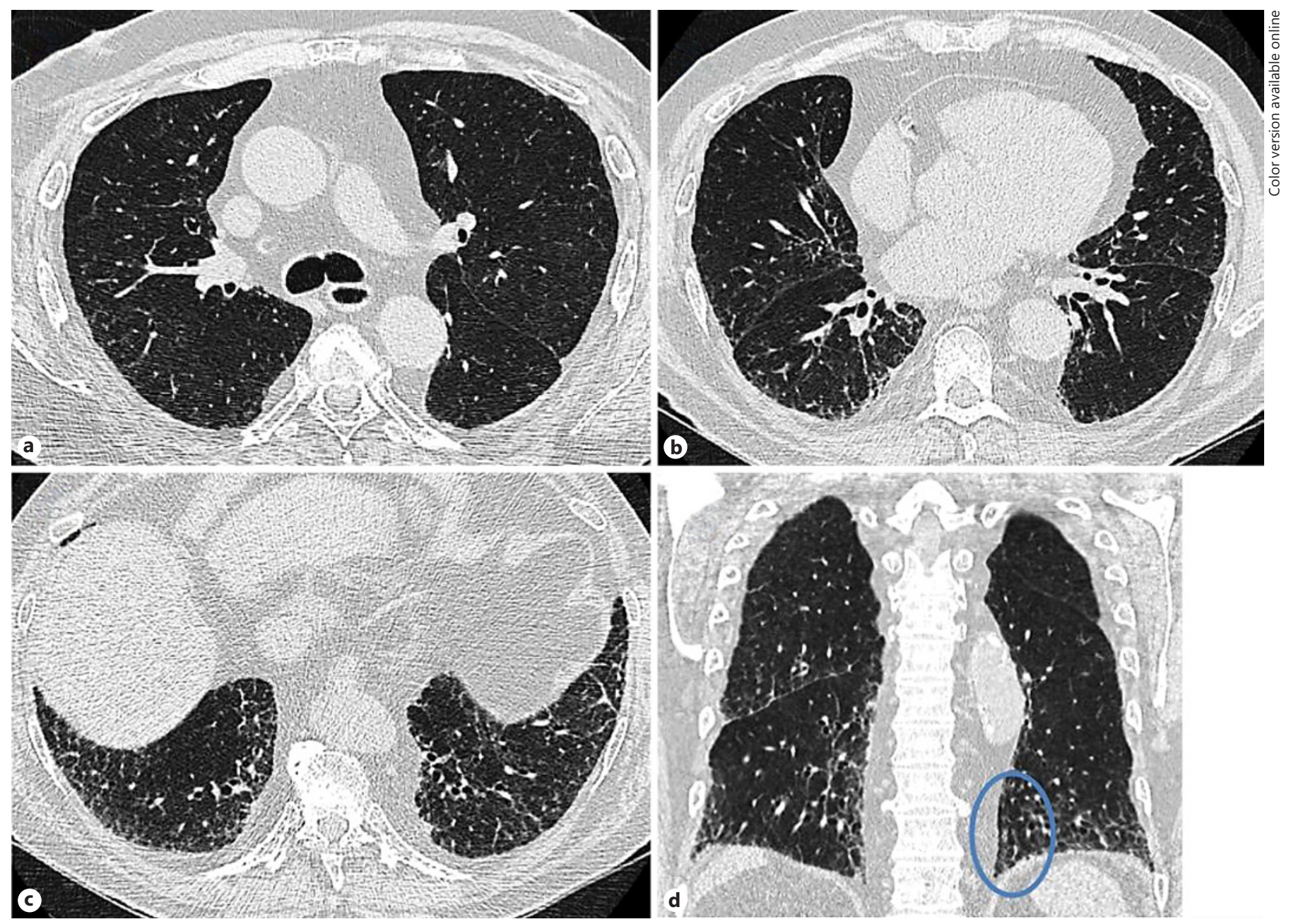

Fig. 2. Probable UIP pattern on HRCT: (a-d) the CT images show increased basal-predominant subpleural reticulation with peripheral traction bronchiectasis (circle in $\mathbf{d}$ ) but without honeycombing.

ticular abnormalities [46]. "Pure" GGO is NOT a typical feature of UIP, and its presence in a patient with IPF could indicate an acute exacerbation $[47,48]$. In contrast, GGO superimposed on fine reticular abnormalities indicates fibrosis and may be seen in patients with IPF. The combination with traction bronchiectasis/bronchiolectasis helps in the differentiation of these 2 patterns [42].

\subsection{HRCT Patterns}

We recommend using the 4 diagnostic categories described in the publication of the Fleischner Society. These categories are "UIP pattern," "probable UIP pattern," "pattern indeterminate for UIP," and "alternative pattern” (Fig. 1-4) [32].

\subsection{UIP Pattern}

UIP is the hallmark radiological pattern of IPF. Honeycombing is a distinguishing feature of UIP and must be present for a definite HRCT diagnosis of UIP to be made. It can be seen with or without peripheral traction bronchiectasis/bronchiolectasis. The typical distribution of UIP is subpleural with basal predominance although upper lobe involvement is common. In some cases, the craniocaudal distribution of UIP may be relatively uniform [49]. Asymmetric lung involvement can be seen in up to $25 \%$ of cases [50]. Several studies have documented that the positive predictive value of a UIP pattern on HRCT is between 90 and $100 \%$. Therefore, a UIP pattern on HRCT is a highly accurate predictor of a UIP pattern in surgical lung biopsy $[51,52]$. Other studies have demonstrated 


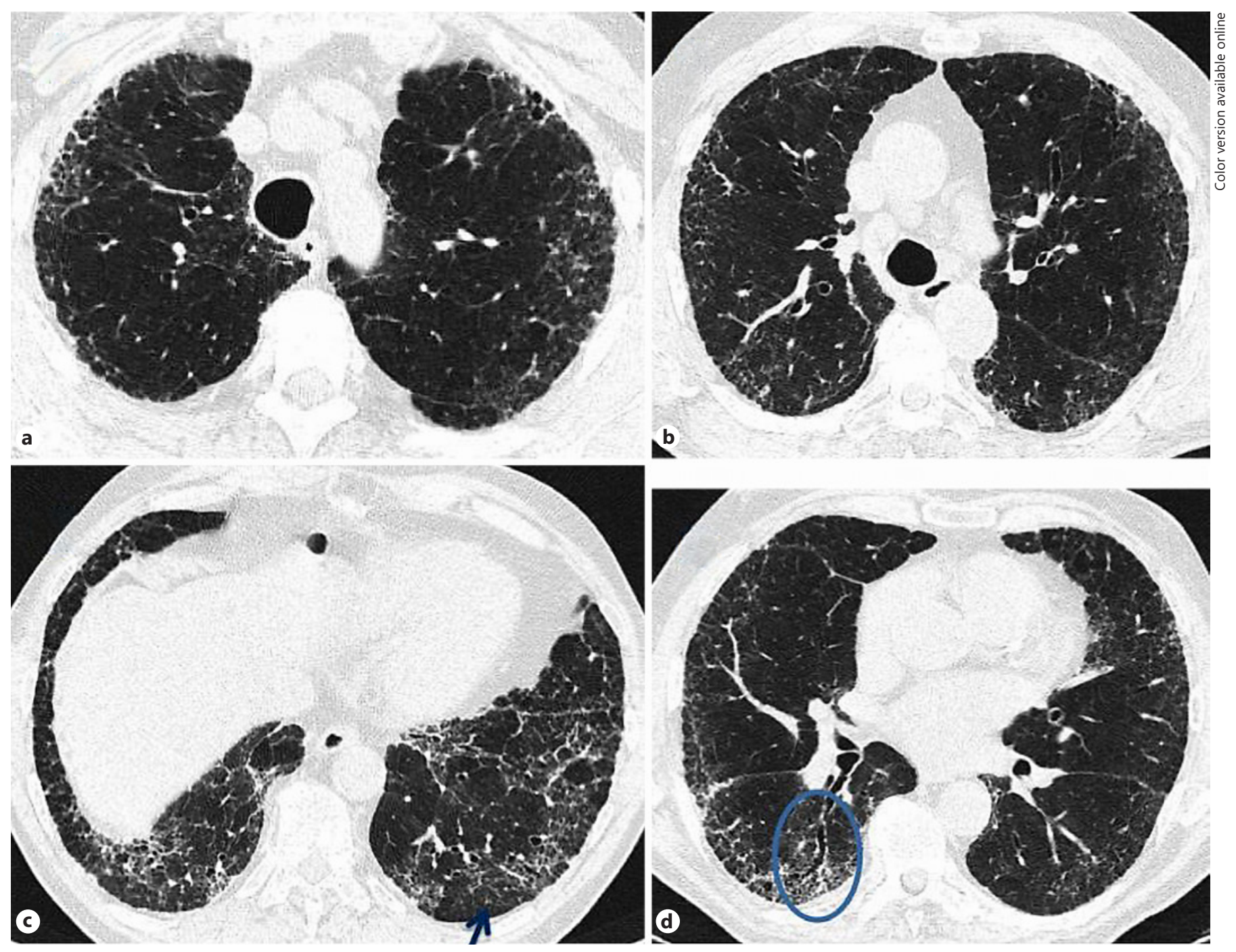

Fig. 3. Indeterminate for UIP: (a-d) the CT images show increased reticulation with traction bronchiectasis (circle), without honeycombing. There is no recognizable craniocaudal gradient. The findings are not typical of a UIP pattern due to the mosaic attenuation and subpleural sparing in the recess (arrow).

that a minority (approx. 30\%) of patients with histopathologically confirmed UIP pattern did not meet the HRCT criteria for UIP pattern [53].

Mild mediastinal lymph node enlargement may be present [54]. GGO may be present but is usually less widespread than reticulation. It is usually superimposed by fine reticulation. Rarely, small ossified nodules within areas of fibrosis are observed, and these are more common in patients with UIP than in those with other fibrotic lung diseases $(29 \%)[55,56]$. Patients with UIP pattern may, in addition, have some features of idiopathic pleuroparenchymal fibroelastosis at the lung apices [57]. However, there is no clear limit to the proportion of each pattern, and these cases should be regarded as UIP/IPF.

IPF patients can present for the first time with acute exacerbation, which makes a CT diagnosis more difficult. Combined features of emphysema are possible in the context of CPFE [58].

\subsection{Probable UIP Pattern}

In the earlier S2K guideline, an HRCT pattern of subpleural, basal-predominant reticular abnormalities without honeycombing was classified in the HRCT diagnostic 


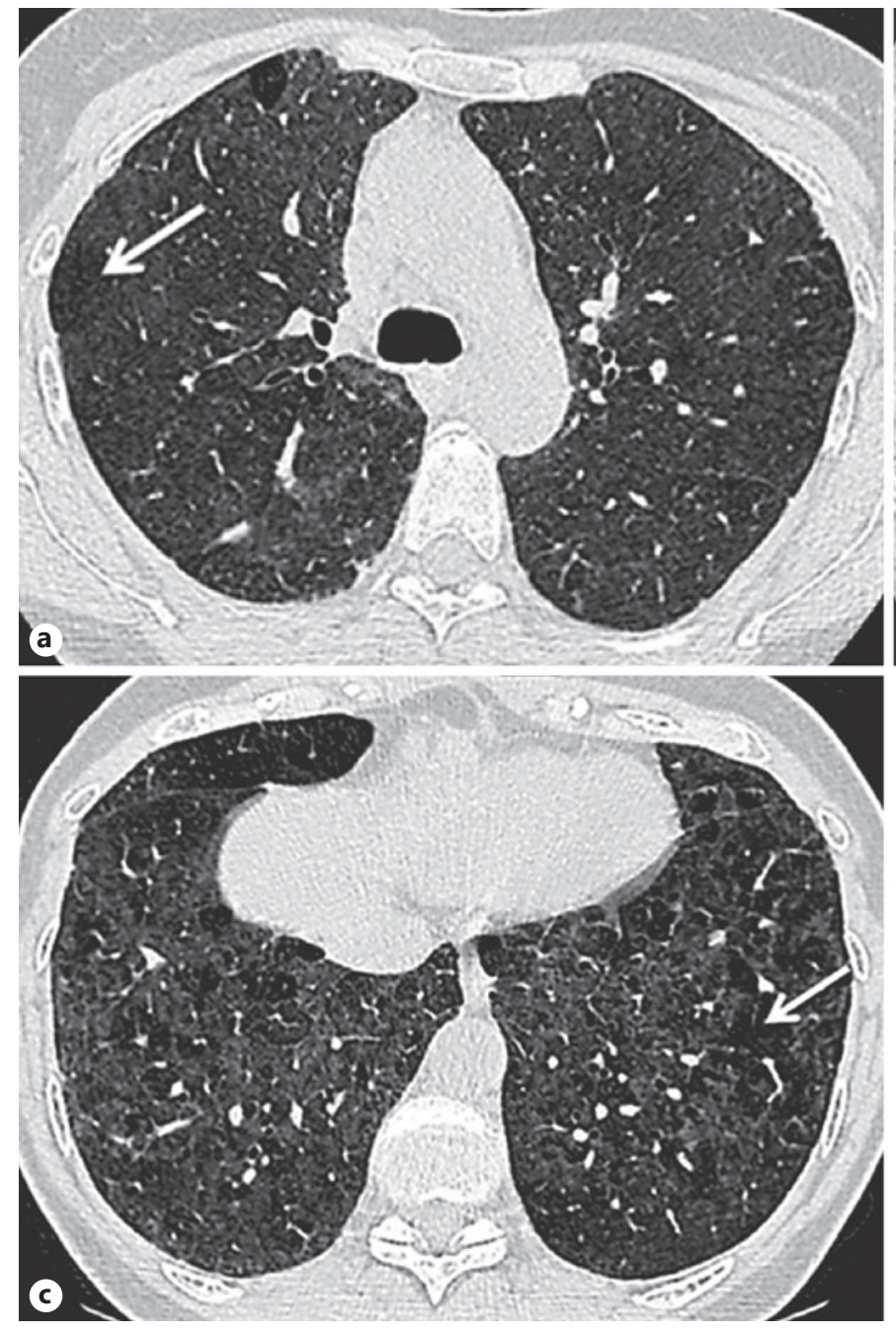

category of "possible UIP." Since then, several studies have reported that patients with a "possible UIP" pattern on HRCT as per S2K guidelines are highly likely to have a histopathological UIP pattern, despite the absence of honeycombing in $\mathrm{x}$-ray [59]. Therefore, subpleural, basal-predominant reticular abnormalities with peripheral traction bronchiectasis or bronchiolectasis should be regarded as "probable UIP." As with a UIP pattern, GGO may be present in probable UIP, but it is not a dominant feature [60].

\subsection{Indeterminate for UIP}

Atypical HRCT features frequently accompany a histopathological pattern of UIP (in about $30 \%$ of cases) [61]. The category "indeterminate for UIP pattern" should, therefore, be assigned when the HRCT shows features of pulmonary fibrosis without meeting the criteria of typical or probable UIP, and an alternative diagnosis

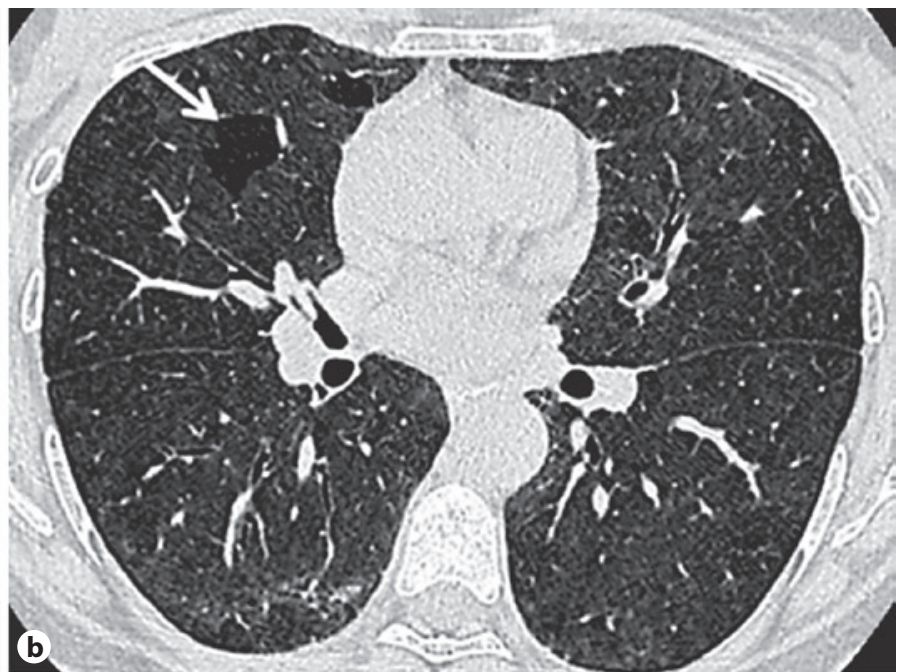

Fig. 4. a-c Alternative pattern: the CT images show extensive diffuse GGO. Isolated secondary nodules show mosaic attenuation (arrows). The pattern is consistent with that of HP.

cannot be explicitly suggested. This category also includes a subset of patients with minimal subpleural GGO or reticulation without obvious CT features of fibrosis, for these patients there is a suspicion that early UIP or probable UIP is present. In these cases, prone CT views should be used to confirm that the subpleural opacities do not represent hypostasis/position-dependent atelectasis of the lung parenchyma.

\subsection{Alternative Pattern}

There may be cases where IPF is clinically suspected, but the HRCT pattern suggests an alternative diagnosis. Examples include upper lobe-predominant peribronchial fibrosis with profuse mosaic attenuation of the lung parenchyma suggestive of $\mathrm{HP}$, perihilar fibrotic retraction in sarcoidosis, or extensive GGO with subpleural sparing in fibrotic nonspecific interstitial pneumonia (NSIP). 

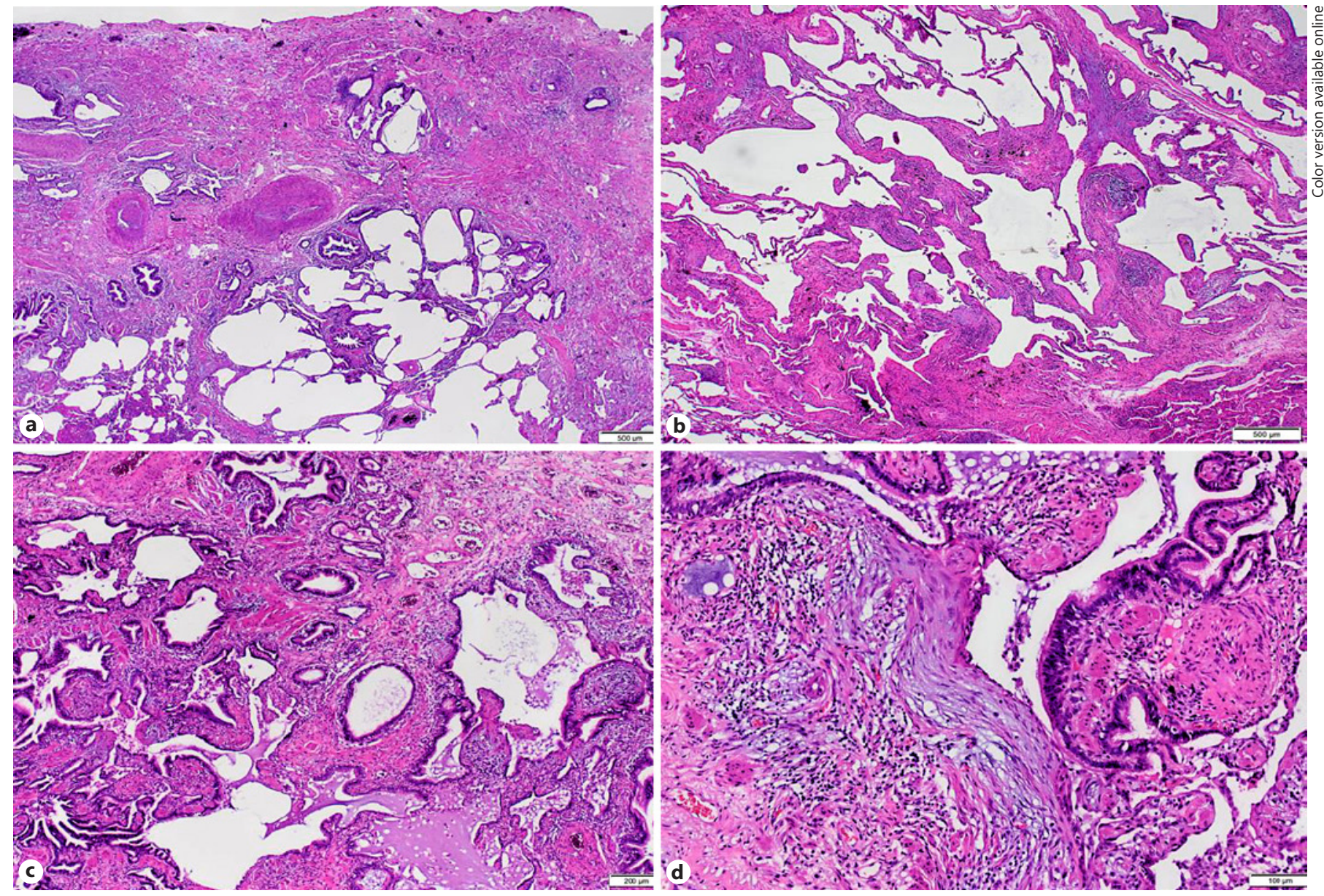

Fig. 5. UIP - histological patterns and features. Characteristic histopathological features of the UIP pattern include the following: prominent, heterogeneous fibrosis with extensive involvement of the visceral pleura and prominent smooth muscle (myogenic) metaplasia (a); abrupt transition between remodeled lung parenchyma and lung parenchyma (not yet) affected by fibrotic remod-

eling with inconspicuous alveolar septa (b); severe architectural distortion of lung parenchyma with extensive type II pneumocyte hyperplasia and mucus retention (c); defined subepithelial myofibroblast accumulation, so-called fibroblastic focus, adjacent to stroma with interspersed inflammation (d).

Occasionally, the HRCT presentation may be that of a typical, probable, or indeterminate UIP pattern, while ancillary findings suggest an alternative diagnosis. In such situations, an alternative diagnosis to IPF should be reconsidered.

\subsection{CT Findings in Acute Exacerbation}

Patients with acute IPF exacerbation have bilateral GGO of the lung parenchyma with or without consolidation on a background of pulmonary fibrosis. In the absence of a previous HRCT study, bilateral GGO and/or consolidation with additional UIP pattern is highly suggestive of an acute exacerbation and can be used to confirm underlying IPF diagnosis in the appropriate context.

\section{Diagnostic Imaging Recommendations}

Volumetric, high-resolution, non-contrast, inspiratory CT images shall be acquired for all patients with suspected IPF, with patients in supine position. Additional sequential HRCT slices in expiration should be acquired if a disease of the small airways is suspected (e.g., bronchiolitis and HP). Findings can be supplemented with prone scans. An existing CT that does not meet the above quality criteria shall not be used for diagnostic purposes.

We recommend using 4 HRCT diagnostic categories as described in the publication of the Fleischner Society [32]. These categories are "UIP pattern," "probable UIP pattern," "pattern indeterminate for UIP," and "alterna- 
Table 2. HRCT pattern

\begin{tabular}{|c|c|c|c|c|}
\hline & UIP & Probable UIP & Indeterminate for UIP & Alternative pattern \\
\hline Distribution & $\begin{array}{l}\text { Subpleural and basal } \\
\text { predominant; distribution } \\
\text { is often heterogeneous } \\
\text { and asymmetric }\end{array}$ & $\begin{array}{l}\text { Subpleural and basal } \\
\text { predominant; } \\
\text { distribution is often } \\
\text { heterogeneous and } \\
\text { asymmetric }\end{array}$ & Subpleural predominance & $\begin{array}{l}\text { Peribronchovascular or } \\
\text { perilymphatic distribution } \\
\text { Upper or mid-lung predominance }\end{array}$ \\
\hline Features & $\begin{array}{l}\text { Honeycombing with or } \\
\text { without peripheral } \\
\text { traction bronchiectasis or } \\
\text { bronchiolectasis }\end{array}$ & $\begin{array}{l}\text { Reticular pattern with } \\
\text { peripheral traction } \\
\text { bronchiectasis or } \\
\text { Bronchiolectasis }\end{array}$ & $\begin{array}{l}\text { Subtle reticulation with or without GGO } \\
\text { CT features and/or distribution of lung } \\
\text { fibrosis that do not suggest any specific } \\
\text { etiology }\end{array}$ & $\begin{array}{l}\text { CT features suggestive of another } \\
\text { diagnosis } \\
\text { Cystic lung disease } \\
\text { Marked mosaic attenuation } \\
\text { Predominant GGO } \\
\text { Profuse micronodules } \\
\text { Nodules } \\
\text { Consolidation } \\
\text { Other features } \\
\text { Pleural plaques } \\
\text { Dilated esophagus } \\
\text { (CTD) } \\
\text { Distal clavicular erosions (RA) } \\
\text { Extensive lymph node enlargement } \\
\text { Pleural effusions }\end{array}$ \\
\hline
\end{tabular}

HRCT, high-resolution computed tomography; GGO, ground-glass opacity; CTD, connective tissue disease; RA, rheumatoid arthritis.

tive pattern." Radiology reports shall include an adequate description and apply the guideline-specific diagnostic criteria and classification system.

\section{Histopathology Aspects}

In (fibrosing) ILDs, a large number of noxious agents or triggers and pathological stimuli correspond to only a limited number of morphological injury patterns, each with significant overlap. Therefore, a correct diagnostic classification is only possible based on a synoptic consideration of clinical, radiological, and histopathological findings $[62,63]$.

The histological hallmark of UIP is a patchy mix of interstitial fibrosis with extensive remodeling of the original pulmonary architecture and areas with inconspicuous or only slightly remodeled parenchyma. As with most fibrosing ILDs, these changes are best identified on low magnification images (Fig. 5). Parenchymal remodeling begins in the subpleural and basal lung areas so that - especially in the early stages of disease - the subpleural compartments are most frequently affected in UIP. As the interstitial myofibroblast-driven remodeling progresses, also the centrilobular regions become increasingly affected. In addition to the heterogeneous distribution of fi- brosed and non-fibrosed areas in terms of time and space, and the marked pleural/subpleural, as well as paraseptal fibrosis, an increasing number of honeycomb-like cysts with epithelial metaplasia (also known as "microscopic honeycombing"), accumulations of secretion and inflammatory infiltrates, are seen in the scarred areas. In most cases, this is associated with prominent type II pneumocyte hyperplasia and a pronounced so-called myogenic metaplasia with prominent interstitial smooth-muscle metaplasia and mostly mild chronic, occasionally even florid inflammation. Fatty tissue metaplasia or heterogeneous ossification can also occur. In addition, a varying number of localized proliferating myofibroblasts with cube-like epithelial lining, known as fibroblast foci and very typical for a fully developed UIP, can be found, especially in the transitional zone between the fibrotic and non-fibrotic areas $[63,64]$. However, the detection of fibroblast foci is by no means specific for UIP.

Annotation: it should be noted, however, that there is currently no generally accepted definition of fibroblast foci and that similar lesions are seen in a large number of fibrotic lung diseases.

Interstitial inflammation in UIP can range from discrete to prominent. In the absence of a granulocytic inflammatory component, the detection of prominent lymphofollicular aggregates in patients with otherwise typical 
Table 3. Histopathology patterns and features

\begin{tabular}{llll}
\hline UIP & Probable UIP & Indeterminate for UIP & Alternative pattern \\
\hline $\begin{array}{l}\text { Dense fibrosis with } \\
\text { architectural distortion } \\
\begin{array}{l}\text { (i.e., destructive scarring } \\
\text { and/or honeycombing) }\end{array}\end{array}$ & $\begin{array}{l}\text { Some histologic features from } \\
\text { column 1 are present but to an } \\
\text { extent that precludes a definite } \\
\text { diagnosis of UIP/IPF and }\end{array}$ & $\begin{array}{l}\text { Fibrosis with or without architectural } \\
\text { distortion, with features favoring either a } \\
\text { pattern other than UIP or features favoring UIP } \\
\text { secondary to another cause }\end{array}$ & $\begin{array}{l}\text { Features of other histologic patterns } \\
\text { of IIPs (e.g., absence of fibroblast } \\
\text { foci or loose fibrosis) in all biopsies }\end{array}$ \\
$\begin{array}{llll}\text { Predominant subpleural } \\
\text { and/or paraseptal } \\
\text { distribution of fibrosis }\end{array}$ & $\begin{array}{l}\text { Absence of features to suggest an } \\
\text { alternative diagnosis, or } \\
\text { honeycombing only }\end{array}$ & $\begin{array}{l}\text { Some histologic features from column 1, but } \\
\text { with other features suggesting an alternative } \\
\text { diagnosis }{ }^{2}\end{array}$ & $\begin{array}{l}\text { Histologic findings indicative of } \\
\text { other diseases (e.g., Hypersensitivity } \\
\text { pneumonitis, langerhans cell } \\
\text { histiocytosis, sarcoidosis, LAM) }\end{array}$ \\
\hline & & $\begin{array}{l}\text { Patchy involvement of lung } \\
\text { parenchyma by fibrosis }\end{array}$ & $\begin{array}{l}\text { Fibroblast foci } \\
\text { Absence of features to suggest an } \\
\text { alternate diagnosis }\end{array}$ \\
\hline
\end{tabular}

IIP, idiopathic interstitial pneumonia; IPF, idiopathic pulmonary fibrosis; LAM, lymphangioleiomyomatosis; UIP, usual interstitial pneumonia. ${ }^{1}$ Granulomas, hyaline membranes (other than those associated with acute IPF, exacerbation, which may be the presenting manifestation in some patients), prominent, airway-centered changes, areas of prominent interstitial inflammation without associated fibrosis, marked chronic fibrosing pleuritis, organizing pneumonia. Such features may not be easily seen by the untrained eye and often need to be specifically sought. ${ }^{2}$ Changes that should raise concerns about the likelihood of an alternative diagnosis include inflammatory infiltrates away from areas of honeycombing, prominent lymphoid hyperplasia.

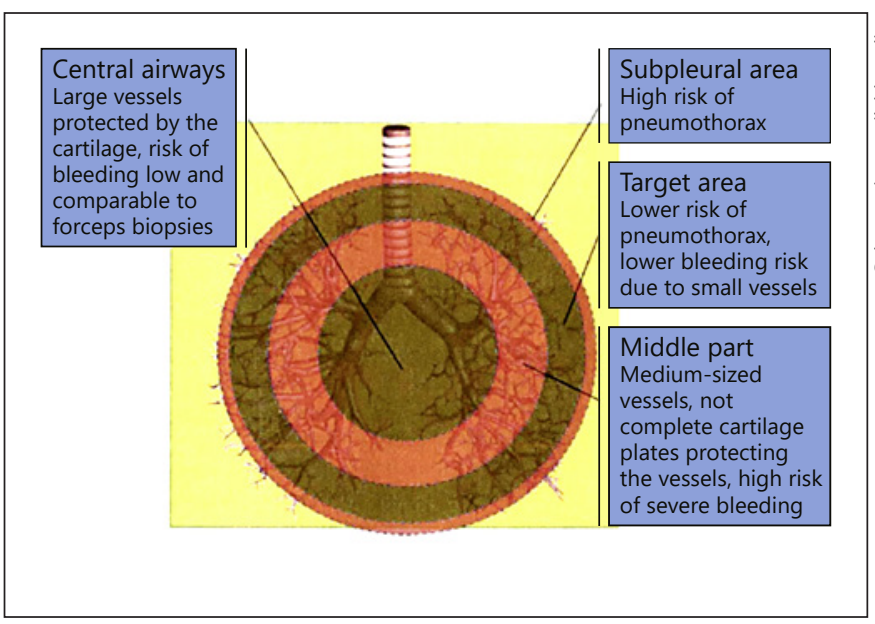

Fig. 6. Mathodological and safety aspects of TBLC (from Hetzel et al. [143] with permission).

UIP the pulmonary involvement of a primarily extrapulmonary condition, for example, a rheumatic disease, should be considered and/or discussed.

In line with the ATS/ERS criteria, the histopathological patterns shall be categorized as "UIP," "probable UIP," "indeterminate for UIP," or "alternative pattern" (see Table 3) [5]. The categories are based on defined positive and/or negative criteria. The categories as presented in this document have been modified/updated against the previous ATS/ERS statement of 2011 [62], and the S2K guideline based on it [1]. Given the high level of evidence for the specificity of HRCT in detecting a UIP pattern, lung biopsy continues not to be mandatory if a UIP pattern is identified on HRCT. Therefore, if an appropriate clinical context is met, an HRCT pattern of UIP is sufficient to diagnose IPF (Fig. 6, 7).

When assessing tissue samples, and before conclusively interpreting the identified morphological histopathological changes, the elements of parenchymal remodeling present should always be listed and the compartments affected by remodeling (affected lung areas, bronchi/bronchioles, alveolar space, interstitium, arterial and venous vascular branches, etc.) systematically described, to comprehensively document the intensity and spatial arrangement of such remodeling processes. This allows for a better comparison of a patient's findings over time or, if necessary, enables other institutions to reclassify the patient based on written documentation.

Also to be taken into account in this context are the inherent problems associated with different types of sampling, for example, the inherent inability to address pleural involvement in fibrotic remodeling in transbronchial biopsies, which by their very nature do not include the visceral pleura or the lung parenchyma next to it. 


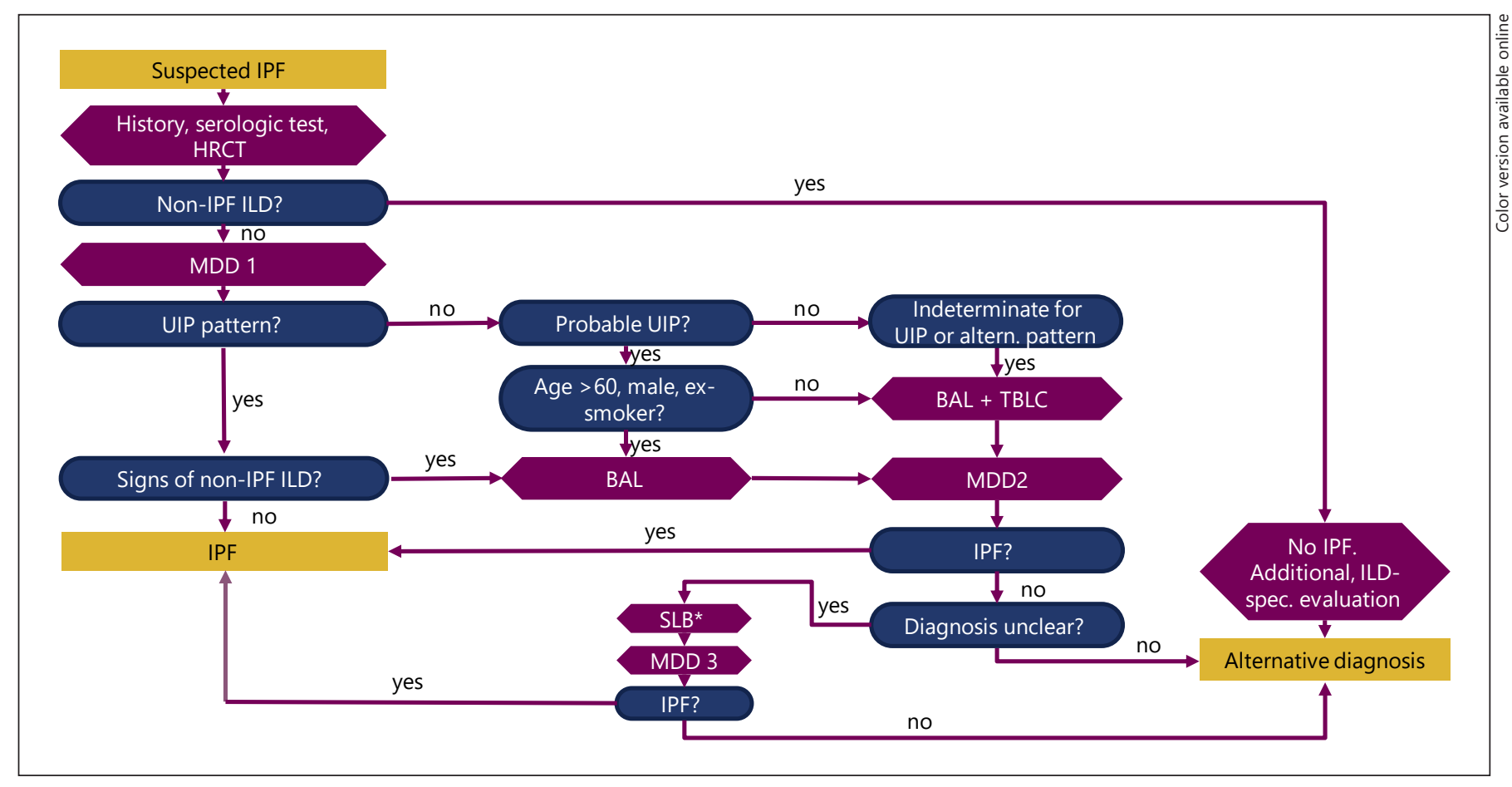

Fig. 7. Flow chart: process for diagnosing ILD. ${ }^{*}$ Or TBLC if not already done and not contraindicated: the decision for surgical lung biopsy is to be based on the clinical condition of the respective patient. It is not indicated in patients at high risk of intra-, peri-, or postprocedural complications (for example, severe hypoxemia at rest and/or severe pulmonary hypertension and/or diffusing capacity of $<40 \%$ [151].

If the histomorphological evaluation has to be done irrespective of imaging and clinical information (e.g., exposure to extrinsic noxious agents, drugs, presence of CTD, HP, or pneumoconioses), that is, if this information is not available at the time of diagnosis, the final diagnosis should only be made after clinicians, radiologists, and pathologists experienced in ILD diagnosis have assessed all the findings in the context of a multidisciplinary case conference (algorithm in Fig. 7), including secondary germinal centers, and a distinctly bronchiolocentric distribution pattern that can go along with extensive peribronchiolar metaplasia.

\section{Histopathology Recommendation}

The histopathology report shall include an adequate systematic description of alterations present and apply the diagnostic criteria and classification system of the guideline.

\subsection{Diagnostic Procedure}

The following questions refer explicitly to patients with clinically suspected IPF. Typically, this includes mostly male patients $>60$ years of age with symptomatic or asymptomatic bilateral ILD on imaging and bibasilar inspiratory crackles on auscultation. The questions should, however, also be applied to younger patients (4060 years of age), especially for patients with a potential risk for familial IPF, as these patients may present with similar symptoms and clinical features. The following recommendations refer to patients with morphological changes suggestive of ILD on HRCT.

\section{Clinically Relevant Questions}

Question 1: should patients with newly detected ILD of unknown cause who are clinically suspected of having IPF undergo a detailed, prompted history of (previous) medication use and inhalational exposures at home and at work, to exclude potential causes of the ILD? 
Table 4. Advanced serological testing: recommended additional serological testing in case of clinical suspicion/positive ANA and screening test

\begin{tabular}{|c|c|}
\hline Suspected diagnosis & Serological tests \\
\hline Myositis & $\begin{array}{l}\text { Muscle enzymes (Cr phosphokinase, myoglobin, and aldolase), myositis-specific autoantibodies: } \\
\text { antisynthetase antibodies (anti-jo-1 and others), anti-MDA5, anti-mi-2, anti-NXP2, anti-TIF1- } \gamma \text {, } \\
\text { anti-SRP, anti-HMGCR, and anti-SAE. Myositis-associated autoantibodies: anti-PM/Scl75, } \\
\text { anti-PM/Scl100, anti-ku, and anti-U1RNP }\end{array}$ \\
\hline Systemic sclerosis & $\begin{array}{l}\text { Anti-Scl-70/topoisomerase-1, anti-centromere, anti-RNA polymerase III, anti-U1RNP, anti-Th/To, } \\
\text { anti-PMScl, U3 RNP (fibrillarin), and anti-ku }\end{array}$ \\
\hline Sjögren's syndrome & Anti-SSA/Ro and anti-SSB/La \\
\hline Vasculitis & ANCA \\
\hline \multicolumn{2}{|c|}{$\begin{array}{l}\text { HMGCR, 3-hydroxy-3-methylglutaryl-CoA reductase; MDA5, melanoma differentiation-associated gene } 5 \text { antibody; NXP2, nuclear } \\
\text { matrix protein 2; PM/Scl75, polymyositis/scleroderma 75; PM/Scl100, polymyositis/scleroderma 100; RNP, ribonucleoprotein; SRP, } \\
\text { signal recognition particle; TIF1- } \gamma \text {, transcriptional intermediary factor } 1 \text { gamma; ANCA, anti-neutrophil cytoplasmic antibodies. }\end{array}$} \\
\hline
\end{tabular}

A detailed, complete review of (prior) medication use and inhalational exposures in the patient-specific environment provides an indispensable basis for identifying or excluding potential causes of the ILD (e.g., HP, pneumoconiosis, and drug toxicity). A retrospective study of 1,084 patients with newly diagnosed ILD of unknown cause shows that $47 \%$ of the patients could be diagnosed with HP on detailed assessment. The data show how important it is to accurately record inhalational triggers [65]. The guideline group agrees that identifying and removing potential causative environmental factors can have a positive impact on the course of the disease. Questionnaires can be useful in clinical practice to make a standardized assessment of exposures at home, at work, and in frequently visited places [65-67]. For German-speaking countries, the guideline panel recommends using the recently published questionnaire of Kreuter et al. [68].

Examples of relevant exposures include mold, birds including down feathers, other animals, metal dusts (e.g., brass, lead, and steel), wood dust or vegetable dust (e.g., pine trees), dust from stone polishing and cutting, medicines, cigarette smoke, current or recent occupation (e.g., hairdresser and dental technician), and hobbies [69-75]. Serological screening tests (for specific IgG antibodies) can help to identify a potentially triggering agent for HP that was not suspected based on the patient's clinical history. However, serum antibody tests are not standardized, and their specificity and sensitivity for the diagnosis of $\mathrm{HP}$ is unknown. Evidence of sensitization gained from in vitro testing alone is not indicative of disease. Conversely, negative antibody testing does not exclude HP. It is often impossible to fully prove a causal relationship between ILD and exposure.

Recommendation regarding History of Exposure

For patients with recently identified ILD of apparently unknown cause, who clinically meet the criteria of a presumptive IPF diagnosis, a detailed and complete history of both medication use and inhalational environmental exposure shall be obtained in a standardized format to exclude potential causes of ILD.

\section{Question 2: should patients with newly detected ILD} of unknown cause who are clinically suspected of having IPF undergo serological testing to exclude or diagnose an underlying autoimmune disorder?

No reliable data are available on the role of serological screening in patients suspected of having IPF. However, the diagnosis of IPF requires exclusion of other causes of ILD. CTD-associated ILDs (or collagenosis-associated ILDs) which may be associated with a UIP pattern, constitute a differential diagnosis. There are patients with CTD in whom the ILD, that is, the lung involvement, is the first, dominant, or only feature. Criteria have been proposed for defining patients with clinically, serologically, and morphologically suspected autoimmune disease as having "interstitial pneumonia with autoimmune features"; this definition is currently in the process of being scientifically and clinically validated [76]. Serological screening is, therefore, recommended in all patients with newly identified ILD even if no other signs and symptoms of a CTD are found. Serological screening should include 
testing for CRP, sedimentation rate, antinuclear antibodies (titers and interpretation of the fluorescence pattern, ENA), rheumatoid factor, myositis panel, anti-cyclic citrullinated peptide, and anti-neutrophil cytoplasmic antibodies [77]. Additional detailed serological testing is done on a case by case basis (see Table 4 ).

Patients with suspected CTD and those with characteristics atypical for IPF (e.g., female, $<60$ years), should be seen by a rheumatologist for further diagnostic evaluation (e.g., capillary microscopy). However, the guideline panelists do not generally recommend that all patients suspected of having IPF but who otherwise have no serological signs of systemic disease should be seen by a rheumatologist. By analogy, specific IgG antibodies (precipitins) against antigens known to be frequent triggers of $\mathrm{HP}$ can be measured; however, their significance is difficult to assess.

Recommendation regarding Serological Tests

For patients with newly identified ILD of yet unknown cause who are clinically suspected of having IPF, serological testing shall generally be performed to identify a CTD as a potential cause of ILD.

Question 3: should patients with newly detected ILD of unknown cause who are clinically suspected of having IPF undergo cellular analysis of their BAL fluid?

The systematic literature search for the international guideline yielded 2,492 titles. However, no studies were identified that (1) compared clinical outcomes between patients who underwent BAL cellular analysis to those who did not or (2) reported the test characteristics of BAL cellular analysis for distinguishing IPF from other ILDs. Therefore, studies comparing BAL cell-type distribution across different ILDs were included; 8 of 14 studies were selected for analysis [78-85].

Annotation: the limitation of the international guideline is that only studies conducted since 2010 were taken into account. As a result, many older papers on BAL comparing the differential cytology of major lung diseases were not considered for the international guideline.

The included studies determined the percentage of neutrophils [78-83, 85], alveolar macrophages [78-82, 85], lymphocytes [78-85], and eosinophils [78, 80-83, $85]$, as well as the CD4/CD8 ratio $[78,80,82,83]$ in BAL. The data of IPF patients were then compared with those from patients with other ILD diagnoses such as HP $[78,79,83]$, sarcoidosis [78, 82, 83], idiopathic NSIP $[78,80,83-85]$, cryptogenic organizing pneumonia (COP) [78-80, 83], eosinophilic pneumonia [78], RB-
ILD [79], and LIP [79]. Most studies reported the mean cell-type proportions, while some reported medians. The analysis compared the mean difference (MD) of the mean values between IPF and other groups of diseases; studies providing the median were, therefore, no longer considered.

\section{Neutrophil Percentage}

The percentage in healthy individuals is $\leq 3 \%$. Patients with IPF had a mean value ranging from 5.9 to $22.1 \%$. This was higher than that in HP (MD +4.84\%; 95\% CI, +1.70 to $+7.98 \%$ ), cellular NSIP (MD $+3.40 \%$; $95 \%$ CI, 0.33 to $+6.47 \%$ ), eosinophilic pneumonia ( $\mathrm{MD}+16.8 \%$; $95 \% \mathrm{CI},+1.96$ to $+31.62 \%)$, RB-ILD (MD + 11.8\%; $95 \%$ $\mathrm{CI},+9.04$ to $+14.56 \%)$, and LIP (MD + 7.40\%; 95\% CI, +3.3 to $+11.5 \%)$. No differences were found when compared with patients with fibrotic NSIP, COP, or sarcoidosis.

\section{Eosinophil Percentage}

The percentage in healthy individuals is $\leq 1 \%$. Patients with IPF had a mean value ranging from 2.39 to $7.5 \%$. This was lower than that in patients with eosinophilic pneumonia (MD $-48.94 \%$; $95 \% \mathrm{CI},-62.58$ to $-35.30 \%$ ). No differences were found when compared with patients with NSIP, HP, COP, sarcoidosis, RB-ILD, or LIP.

\section{Lymphocyte Percentage}

The percentage in healthy individuals is $10-15 \%$. Patients with IPF had a mean lymphocyte percentage ranging between 7.2 and $26.7 \%$. This was lower than that in patients with NSIP (MD -26.0\%; 95\% CI, -33.62 to $18.38 \%$ ), sarcoidosis ( $\mathrm{MD}-14.87 \%$; $95 \% \mathrm{CI},-25.09$ to $-4.65 \%)$, $\mathrm{COP}(\mathrm{MD}-31.43 \%$; $95 \% \mathrm{CI},-38.78$ to $-24.08 \%)$, and LIP (MD $-43.20 ; 95 \% \mathrm{CI},-48.83$ to $-37.7 \%$ ). The lymphocyte percentage was higher in patients with IPF than in those with RB-ILD (MD 3.03\%; 95\% CI, +1.04 to $+5.56 \%)$. No differences were found when compared with patients with HP or eosinophilic pneumonia.

Annotation: surprisingly, no difference was found with regard to HP. This was due to the selection of publications and the exclusion of older articles/studies (published before 2010) in the literature search. In the case of HP, only 2 studies were considered in the international guideline: a study by Schildge et al. [79], which found a lymphocyte percentage of $51.4 \%$ in HP and only $9.1 \%$ in IPF, and a study by Lee et al. [78] with a very small number of cases, in which the lymphocyte percentage in HP $(n=9)$ was only $19.9 \%$, and in "UIP" $(n=15)$ was surprisingly high (21.2\%). A study by Schildge et al. [86] from 
2007, which was not considered, compared large numbers of patients with sarcoidosis, IPF, COP, CTD-ILD, RB-ILD, and HP. Here, the resulting mean lymphocyte value was $54.5 \%$ for HP versus only $10.2 \%$ for IPF.

\section{CD4/CD8 Ratio}

Healthy individuals have a ratio of $0.9-2.5$. Patients with IPF had a mean ratio of 1.4-7.2. This was lower than that in patients with sarcoidosis (MD $-5.49 ; 95 \% \mathrm{CI}$, -8.45 to -2.53$)$ and higher than in patients with NSIP ( $\mathrm{MD}+0.9 ; 95 \% \mathrm{CI},+0.43$ to +1.47$)$. No differences were found in comparison to HP, COP, eosinophilic pneumonia, RB-ILD, or LIP.

\section{Evidence Assessment}

The guideline panel had very low confidence in the estimated differences in BAL cell distribution between IPF and other ILDs. This was due to the small number of studies, the low number of cases, and the high variability of the distribution across the different studies. In addition, there was a risk of selection bias due to lack of consecutive patient enrollment, as well as detection bias due to different laboratory methods and bronchoscopy techniques used. The statistically significant differences were small and not considered clinically significant.

\section{Positive Consequences}

Cellular BAL analysis can help differentiate between IPF and a few other ILDs, especially in eosinophilic pneumonia and sarcoidosis.

\section{Negative Consequences}

Although no BAL-associated complications were reported in any of the studies, bronchoscopy is an invasive method that requires time, goes along with potential complications, and is uncomfortable for some patients.

\section{Conclusion}

Despite the very low confidence in the estimated effects, the guideline panel believes that the positive consequences outweigh the negative consequences of cellular BAL analysis in patients without a definite UIP pattern on HRCT. BAL is particularly useful if the radiological differential diagnosis includes eosinophilic pneumonia, sarcoidosis, HP, or infection. If a definite UIP pattern is present, BAL can provide relevant additional diagnostic information if there are concrete signs for an alternative etiology of the disease.
Recommendations regarding $B A L$

All patients with newly detected ILD of unknown cause and clinically suspected IPF shall undergo BAL if no definite UIP pattern is seen on HRCT. If a definite UIP pattern on HRCT is present, BAL can be performed if there are concrete signs of an alternative etiology of the disease.

Question 4: for patients with newly detected ILD of unknown cause who are clinically suspected of having IPF, should surgical lung biopsy (SLB) be performed to ascertain the histopathology diagnosis of UIP pattern?

There are 26 studies [87-112] of patients with unclear ILD in which the diagnostic accuracy of SLB was investigated and a multidisciplinary ILD board was used as the gold standard. In all cases, adequate tissue could be sampled by SLB (11 studies, 918 of 918, 100\%; 95\% CI, 99$100 \%$ ), even though this may not always seem to be the case in a real-world scenario. In most cases, a specific diagnosis can be made based on SLB (26 studies; in 2,338 of 2,651 patients, $88.2 \%$; $95 \%$ CI, 86.9-89.4\%) and only rarely does an ILD remain unclassifiable (26 studies; 313 of 2,651 patients, $11.8 \%$; $95 \%$ CI, 10.6-13.1\%). Considering the final diagnosis of these patients, about one-third are classified as IPF (24 studies; 752 of 2,360 patients, 31.9\%; 95\% CI, 30.0-33.8\%). Other diagnoses, some of which are potentially treatable, include infections, sarcoidosis, HP, eosinophilic pneumonia, LAM, COP, or pulmonary vasculitis.

The overall mortality of elective SLB is low (23 studies; 79 of 2,268 patients, $3.5 \%$; $95 \% \mathrm{CI}, 2.8-4.3 \%$ ), the actual procedure-related mortality even lower (6 studies; 7 of 410 patients, $1.7 \%$; 95\% CI, 0.8-3.5\%). Patient selection and the experience of centers in performing biopsies in some instances result in significant differences in the reported mortality rates. For example, a higher number of surgical lung biopsies per year leads to a decrease in the 30 -day mortality rate $(\mathrm{OR}, 0.84 ; 95 \% \mathrm{CI}, 0.73-0.97 ; p=$ $0.02)$, especially in nonelective procedures (OR, $0.84 ; 95 \%$ CI, $0.69-1.02 ; p=0.08)$ and not so much in elective procedures (OR $0.9495 \%$ CI, 0.74-1.18, $p=0.57$ ) [113].

Additional complications and side effects of SLB include the following:

1. Acute exacerbations (15 studies; $6.1 \%$; 95\% CI, 5.1$7.3 \%)$

2. Bleeding (7 studies; $0.8 \%$; $95 \% \mathrm{CI}, 0.4-1.7 \%$ )

3. Severe bleeding (4 studies; 0.2\%; 95\% CI, 0.04-1.2\%)

4. Prolonged air leak (13 studies; 5.9\%; 95\% CI, 4.8$7.2 \%)$ 
5. Respiratory infection (9 studies; 6.5\%; 95\% CI, 4.6$9.0 \%)$

6. Neuropathic pain (1 study; $4.5 \%$; 95\% CI, 1.6-12.5\%)

7. Delayed wound healing (4 studies; $3.3 \%$; $95 \%$ CI, $2.0-$ $5.4 \%)$.

\section{Summary of Evidence}

It can be assumed that the 1-year mortality rate of IPF is reduced from 8 to $5.5 \%$ by antifibrotic therapy, while the probability of slowing down disease progression increases from 60 to $68 \%$. This would mean that in 1,000 surgical lung biopsies, in which 1,000 adequate tissue samples are obtained, 882 specific diagnoses will be made, and 319 of these patients will be determined to have IPF. Assuming that all receive therapy, the 1-year mortality of these 1,000 patients would be reduced from 26 to 18 patients, and disease progression would slow down in 217 instead of 192 patients. In addition, in many patients, a different, treatable ILD would be detected. However, it should be taken into account that 17 patients would die as a result of the surgical procedure, 61 patients would suffer acute exacerbation, and 65 respiratory infection.

If it appears necessary to confirm the histopathological findings of a UIP pattern in patients suspected of having IPF, the superior accuracy and sensitivity of SLB has to be weighed against its higher procedure-related mortality rate compared to bronchoscopic cryobiopsy, and factors such as the clinician's expertise and the number of procedures conducted at an institution need to be considered. In addition to the criteria to be applied to determine that patients are fit to undergo the procedure, the following contraindications to SLB should be considered for ILD patients:

1. Hypoxemia at rest, $\mathrm{SpO}_{2}<90 \%$ on room air

2. TLCO $<40 \%$ of predicted

3. Severe restriction with TLC $<50 \%$ of predicted

4. Pulmonary hypertension with echocardiographically estimated systolic right ventricular pressure $>40 \mathrm{~mm}$ $\mathrm{Hg}$.

\section{Recommendation regarding $S L B$}

For patients with suspected IPF who have undergone the endoscopic diagnostic procedures but where the diagnosis still remains unclear after the multidisciplinary discussion (MDD), SLB shall be performed.

Question 5: should a transbronchial lung biopsy (forceps) be performed for patients who are suspected of having IPF to ascertain the histopathology diagnosis of UIP pattern?
The systematic literature search conducted in the context of the international guideline yielded 945 titles describing the diagnostic use of traditional transbronchial biopsy (TBB) in patients with ILD. However, no studies that compared clinical outcomes between patients with and without TBB could be identified.

Therefore, studies in which the diagnostic yield of TBB was determined using MDD-based decisions were included. Seven studies were selected in this respect, which included patients with ILDs of unknown cause, including patients with UIP pattern on HRCT [111, 114-119].

The analysis showed that TBB obtained an adequate sample in more than three-quarters of cases (78\%). Among these adequate samples, a specific diagnosis was obtained from almost half (43\%), while $57 \%$ were assessed as unclassifiable. Analyzing all the TBB samples (adequate and inadequate), a specific diagnosis could only be established in about one-third of the cases (36\%). This rate (36\%) corresponds to the diagnostic yield.

A significant limitation of these studies is that patients were not stratified by HRCT pattern. Observed complications included pneumothorax (1 study, 5 of $49,10.2 \%$; 95\% CI, 4.4-21.8\%) and prolonged air leak (1 study; 3 of $49,6.1 \%$; 95\% CI, 2.1-16.5\%). No procedure-related deaths were reported in this specific study.

Annotation: in the opinion of the German guideline group, complication rates cannot reasonably be derived from a study with only 49 patients. Older studies show a 30 -day mortality rate of $0.2 \%$ and a complication rate of $12.8 \%[120]$.

\section{Summary of Evidence}

Using TBB to confirm a strong suspicion of IPF, the ILD remains unclassifiable in about $64 \%$ of patients. A specific diagnosis can be made in $36 \%$ of cases, thus avoiding surgical biopsy.

Positive consequence: surgical biopsy is avoided in $36 \%$ of cases. Negative consequence: a high percentage of patients remain undiagnosed (64\%).

Recommendations regarding Transbronchial Forceps Biopsy

Transbronchial forceps biopsy shall not be performed for the diagnosis of IPF in patients with a definite UIP pattern on HRCT.

No recommendation is provided for patients with other HRCT patterns. 


\section{Assessment}

Very little evidence is available regarding the diagnostic accuracy of TBB in patients with suspected IPF. The panel of experts agreed to advise against TBB in patients with a definite UIP pattern on HRCT. This was based on the low probability of identifying an alternative cause in patients with definite UIP pattern.

Question 6: for patients with newly detected ILD of unknown cause who are clinically suspected of having IPF, is transbronchial lung cryobiopsy (TBLC) a reasonable alternative to SLB to sufficiently ascertain the histopathology diagnosis of UIP pattern?

A systematic literature search for the international guideline yielded no studies comparing the clinical outcome of patients who underwent TLBC to those who underwent SLB. Therefore, studies that analyzed the diagnostic yield were selected. The analysis included 13 of 25 studies $[109,110,116,118,119,121-130]$ that enrolled patients with ILD of unknown cause and did not exclude patients with UIP pattern on HRCT.

The evidence reviewed by the international guideline panel showed that cryobiopsy obtained an adequate sample in the vast majority of cases (10 studies; 720 of 749: 96\%; 95\% CI, 94-97\%). Among those cases where adequate samples were obtained, a specific diagnosis could be established in more than $4 / 5$ of cases (13 studies; 692 of $833: 83 \%$; $95 \% \mathrm{CI}, 80-85 \%$ ); the remaining cases were considered unclassifiable. The diagnostic yield was calculated at $80 \%$ (692 of 862 in 13 studies; 95\% CI, 77-83\%). The overall mortality rate was $2.7 \%$ (7 studies; 15 of 597 , 95\% CI, 1.7-4.3\%); however, some deaths were considered to be related to the patient's underlying disease. Procedure-related mortality was $0.2 \%$ ( 3 studies; 1 of 427 , 95\% CI, 0.04-1.3\%). Additional complications included acute exacerbations ( 3 studies; 1 of $82,1.2 \%$; $95 \%$ CI, $0.2-$ $6.6 \%$ ), bleeding ( 6 studies, 28 of $541,5.2 \%$; 95\% CI, $3.6-$ $7.4 \%$ ) including severe bleeding ( 8 studies; 5 of $674,0.7 \%$; $95 \%$ CI, $0.3-1.7 \%$ ), prolonged air leak (2 studies; 47 of $352,13.4 \%$; 95\% CI, 10.2-17.3\%), and respiratory infections (3 studies; 3 of 409, 0.7\%; 95\% CI, 0.2-2.1\%).

The international guideline's assessment of these data was as follows: for every 1,000 transbronchial cryobiopsy procedures performed, 950 adequate biopsies are obtained, and 790 diagnoses are made (thus avoiding surgical lung biopsies). Accordingly, 210 patients will remain undiagnosed despite TBLC, many of whom would then undergo surgical biopsy. Two patients would die as a result of the TBLC procedure, and 12 would suffer acute exacerbation.
In summary, the international guideline concluded that adequate material is obtained by TBLC in $96 \%(95 \%$ CI, 94-97\%) of patients, from which a definitive diagnosis can be made and SLB avoided in 80\% (95\% CI, 77$83 \%$ ) of cases. Compared with SLB, the rate of associated pulmonary infections is lower in TBLC, and a trend toward less procedural mortality is seen in TBLC. On the other hand, about $20 \%$ of patients ( $95 \%$ CI, $17-23 \%$ ) remain undiagnosed after TBLC, and a higher rate of bleeding or prolonged air leak is observed compared to SLB.

A complementary literature search on TBLC was conducted for the German guideline. It looked for articles/ studies published after the analysis date of the international guideline, and the results were analyzed to provide supplementary information. The search yielded 5 prospective and 8 retrospective studies.

A prospective study compared bleeding-related complications of conventional forceps and cryo-TBB and showed that the rate of clinically relevant bleeding events was higher after cryobiopsy ( 16.2 vs. $4.2 \%, p<0.05)$ although no lethal hemorrhage was seen [131]. Safety aspects were addressed, among others, in a study by Hagmeyer et al. [132] who analyzed optional sequential SLB after TBLC. After initially high morbidity and mortality rates, the procedure was adjusted and significantly lower complication rates were observed.

Two prospective studies analyzed the agreement based on samples obtained by TBLC and by SLB; in both studies, SLB was performed immediately after TBLC in the same session. The study conducted by Romagnoli et al. [133] found agreement of histopathology results in $n=21$ patients enrolled in 2 centers, with $\kappa=0.22$ (95\% CI, 0.010.44 ), concluding that, if SLB had not been performed after TBLC, this would have resulted in a different diagnosis and led to a different treatment in $51 \%$ of cases. In contrast, the multicenter study by Troy et al. [134] found histopathological agreement between TBLC and SLB of $70.8 \%$ in $n=65$ patients, $\kappa=0.70$ (95\% CI, $055-086)$.

Annotation: a critical assessment of these studies concludes that the small number of cases in the Romagnoli study is a significant limitation and can easily result in overestimating the data. When adequate samples were obtained, TBLC contributed to the final MDD diagnosis as frequently as SLB in this study. Against the background of the generally low kappa values, the study by Troy et al. [134] on 65 patients with a kappa of 0.7 shows good agreement between TBLC and SLB.

In addition, a large monocenter cohort of 699 patients showed that at least 2 biopsies should be taken from different sites [135]. The size of the biopsies can differ great- 
ly; according to a retrospective analysis, it ranges from 1.5 to $136.7 \mathrm{~mm}^{2}$, with a mean value of $64.2 \mathrm{~mm}^{2}$, while the diameter can range from 6 to $31 \mathrm{~mm}$, with a mean value of $8.7 \mathrm{~mm}$ [129]. According to a state-of-the-art review, the cryobiopsy sample diameter should be at least $5 \mathrm{~mm}$ to allow for good histological analysis [136]. For the pathologist, cryobiopsy, as compared to TBB, offers the advantage that there are mostly no crushing artifacts.

The control of severe periprocedural hemorrhage can be improved by balloon insertion; the mortality rate was $0.4 \%$ [135]. Real-world data are now also available from Germany [137]. They confirm the published data concerning adequacy of material and diagnostic yield and show, in addition, that cryobiopsy is a feasible option in patients with significantly limited functional status. In the meantime, 30-day mortality data from retrospective monocenter studies have become available. They differ in the respective reports, ranging from $0 \%[137,138]$ to $1.4 \%$ (outpatients) and 5.9\% (inpatients) [135, 139-141]. Regarding the question of TBLC-associated costs versus those of TBB (forceps) and surgical biopsy, a systematic review found a diagnostic yield of 84.4 versus 64.3 versus 91.1\% and cost savings for TBLC of $£ 210$ per patient in the first year and $£ 647$ in subsequent years [142].

\section{Recommendations regarding TBLC}

In summary, the members of the German guideline panel - in contrast to the international guideline - considered TBLC to be preferable over surgical biopsy for the following reasons:

a. When compared to SLB, the diagnostic significance and side effect rates are not considered inferior.

b. The cost of TBLC versus SLB is considered to be lower, and it is expected that far fewer patients will refuse to undergo TBLC than surgery.

c. Patients with significantly advanced ILD can undergo biopsy using TBLC rather than SLB. This may also be the case for elderly patients and possibly for patients with concomitant conditions. It is therefore expected that TBLC will reduce the percentage of unclassifiable ILDs.

The guideline panel, however, recommends that the technical and safety guidelines for cryobiopsy be strictly observed [143]. On a critical note, it should be pointed out that the procedure has not yet been internationally standardized and that only a few, partly contradictory data from controlled studies comparing TBLC versus SLB are available. Further research into this topic is encouraged.

\section{TBLC - Method}

An international expert panel has issued a detailed statement on the technical and safety aspects of TBLC [143], which the German guideline panel seconds with minor modifications.

The following safety aspects and contraindications should be observed when performing TBLC (cf. also Fig. 6):

a. We recommend TBLC to be performed in intubated patients under deep sedation or general anesthesia. Operating rooms for bronchoscopy should be fitted with emergency equipment.

b. We recommend using either a tube for airway management, ideally in combination with a bronchial blocker/Fogarty balloon for the (preventive) control of bleeding, or rigid bronchoscopy. Balloon may not be necessary in this case.

c. We recommend taking at least 2 biopsy samples from at least 2 segments at $1 \mathrm{~cm}$ from the visceral pleura. If possible, cryobiopsy samples with a diameter of no $<5$ $\mathrm{mm}$ should be obtained.

e. We recommend performing the biopsies under fluoroscopic guidance.

f. The site and number of biopsies should be determined in advance and not include areas of severe fibrosis.

g. The interventions should only be performed in centers that are highly experienced in these procedures and in managing-related complications.

The following safety aspects are relevant in TBLC:

a. The main risk factors are pneumothorax and bleeding. Acute exacerbation and death have been described in individual cases.

b. Contraindications for TBLC are bleeding diathesis, continued anticoagulation therapy with thienopyridines, other novel antiplatelet agents, and thrombocytopenia with platelet counts $<50 \times 10^{9} / \mathrm{L}$.

c. Suspected pulmonary hypertension (sPAP $>40 \mathrm{~mm}$ $\mathrm{Hg}$ ) or confirmed pulmonary hypertension (mPAP $\geq 25 \mathrm{~mm} \mathrm{Hg}$ in the right heart catheter), as the risk of bleeding appears to be increased in these cases.

d. FVC $<50 \%$ and/or target TLCO $<30 \%$, depending on clinical presentation

e. Relevant comorbidities (e.g., manifest heart failure and severe emphysema)

Question 7: should patients with newly detected ILD of unknown cause who are clinically suspected of having IPF be diagnosed in the context of a MDD? 
Available data: the systematic search for the international guideline yielded 189 literature references. However, no studies could be identified that (1) compared the clinical results of single-discipline decision-making (SDD; either by a single clinician or by several clinicians from the same discipline) with those of MDD or (2) reported the test characteristics of SDD using MDD as reference standard. For this reason, studies were reviewed that reported agreement between SDD and MDD, and 5 out of 17 studies were selected for analysis [65, 144-147]. Numerous studies had to be excluded as they compared interindividual agreement but did not compare SDD to MDD. In 2017 and 2018, no new papers were published, which met the above criteria and would have needed to be considered for the German version of the guideline.

One study enrolled patients with IPF [65] diagnosed by SDD, and 4 studies enrolled patients with SDD diagnosis of different ILDs, including IPF [144-147]. An MDD diagnosis was consented and compared to the SDD diagnosis. In 3 studies, SDD was done by a single pneumologist $[65,144,145]$, in one by either a single pneumologist or a single internist [146], and in another one by several pathologists [147]. In 3 studies, MDD was done by a pneumologist, a radiologist, and a pathologist $[65,144$, 146]; in one by a radiologist together with a pathologist [65]; and in another one by a pneumologist and a pathologist [147].

Evidence assessment: out of 1,000 diagnostic decisions, agreement between MDD and SDD can be expected in 700 cases. If the MDD is accepted as the reference standard, it must be assumed that the remaining 300 patients are potentially subject to incorrect or delayed treatment or unnecessary diagnostic procedures.

Positive consequences: SDD is the more efficient diagnostic approach compared to the increased time and effort associated with MDD. Negative consequences. if MDD is the accepted reference standard, then SDD demonstrates suboptimal agreement of 70\% (range: 47-87\%).

Conclusion: the guideline panel recommends MDD as the preferred diagnostic approach, as the SDD-related risk of up to $30 \%$ of patients receiving incorrect or late treatment or requiring additional diagnostic testing deemed unacceptable. The panel believes that MDD provides the greatest benefit in patients whose HRCT pattern is probable UIP, indeterminate for UIP, or an alternative diagnosis, or when there are discordant clinical, radiological, and/or histological findings. There has been considerable discussion about what MDD should entail. Until further studies have been completed to optimize MDD, the guideline panel recommends that the group should consist of a pneumologist (and a rheumatologist, as necessary), a radiologist, and a pathologist (if cytology or histology results are available). The mode of operation is to be determined by the clinician. It may be in the form of a face-to-face meeting, telephone or web conference, or by circulating (annotated) documents by e-mail. A face-toface meeting is recommended when discrepant findings need to be discussed.

When measured as proportion, the mean agreement between MDD and SDD was 70\% (47-87\%). When measured using Cohen's kappa coefficient, agreement was only moderate, $\kappa=0.331$ (95\% CI: $0.269-0.392)$. These estimates failed to convince the guideline panel. Contributing factors were as follows: the risk of a systematic recruitment bias by omitting diagnostic problem cases, nonconsecutive recruitment, the inconsistency of estimates, the small study sizes, and potential case selection.

Recommendation regarding $M D D$

The German guideline panel considers the MDD to be the diagnostic gold standard. An initial MDD conference shall be conducted with all available clinical information and HRCT findings to determine the further procedure.

After receiving the results of invasive diagnostic tests, the final diagnosis and further procedure shall then be determined in the context of a second MDD.

Question 8: should patients with newly detected ILD of unknown cause who are clinically suspected of having IPF undergo serum biomarker measurement to corroborate the IPF diagnosis?

The literature search for the international guideline was limited to 4 serum biomarkers based on expert decision: MMP7, SP-D, CCL18, and KL-6. A systematic literature search yielded 429 published articles. However, none of these studies compared clinical outcomes among patients who underwent the measurements of serum biomarkers compared to those who did not.

MMP7: several studies were reviewed that tested the diagnostic value of serum MMP7 concentrations for distinguishing IPF from other ILDs. Two of them were selected $[148,149]$. One study tested the differentiation of IPF from a heterogeneous comparator group that included various other ILDs [148]. In another study, the serum MMP7 levels of IPF patients were compared to a group of patients with NSIP, HP, sarcoidosis, CTD-ILD, and druginduced ILD [149]. Serum MMP7 values had a median sensitivity, specificity, accuracy, and diagnostic odds ratio of $71.7,64.4,68.4$, and $4.7 \%$, respectively. 
Table 5. Combination of HRCT and histopathology patterns in diagnosing IPF

\begin{tabular}{lllll}
\hline Suspected IPF & \multicolumn{2}{l}{ Histopathology } & & \\
\cline { 2 - 4 } & UIP & probable UIP & indeterminate for UIP & alternative diagnosis \\
\hline HRCT pattern & & & & No IPF \\
UIP & IPF & IPF & IPF & No IPF \\
Probable UIP & IPF & IPF & IPF (likely) ${ }^{1 / n o ~ I P F}$ & No IPF \\
Indeterminate for UIP & IPF & IPF (likely) $1 /$ no IPF & Unclassifiable ILD ${ }^{2}$ & No IPF \\
Alternative diagnosis & IPF (likely) $1 /$ no IPF & No IPF & No IPF & \\
\hline
\end{tabular}

HRCT, high-resolution computed tomography; IPF, idiopathic pulmonary fibrosis; UIP, usual interstitial pneumonia; ILD, interstitial lung disease. ${ }^{1}$ IPF is the likely diagnosis if - after excluding alternative causes - any of the following features are present: (a) Moderate to severe traction bronchiectasis and bronchiolectasis (defined as mild traction bronchiectasis/bronchiolectasis involving 4 or more lobes of the lung including lingula, or moderate to severe traction bronchiectasis in 2 or more lobes) in a male patient $>50$ years of age or a female patient $>60$ years of age; (b) Extensive ( $>30 \%$ ) reticulation on HRCT, and $>70$ years of age; (c) Increased neutrophils and/or absence of lymphocytes in bronchoalveolar lavage; (d) The multidisciplinary case discussion agrees on a confident diagnosis of IPF. ${ }^{2}$ Unclassifiable ILD: (a) Without adequate biopsy the diagnosis is unlikely to be IPF; (b) With an adequate biopsy, re-classification to a more specific diagnosis can be made based on multidisciplinary case discussion and/or additional consultation.

SP-D: 16 articles were reviewed that addressed the diagnostic accuracy of SP-D serum levels. Only one study was selected. It showed a sensitivity of $70.0 \%$ and specificity of $65.0 \%$, an accuracy of $68.5 \%$, and a diagnostic odds ratio of 3.1 to distinguish IPF from other types of ILD [148].

CCL18 and KL-6: no valid studies on the diagnostic accuracy of CCL18 and KL-6 serum levels to distinguish IPF from other ILDs were found. The serum levels of these markers do not allow the differentiation between IPF and other ILDs.

\section{Conclusion}

The data published on CCL18 and KL-6 do not suggest that one of these serum biomarkers has a diagnostic value with regard to differentiating IPF from other ILD diseases. For MMP7, 2 studies show a median sensitivity, specificity, accuracy, and diagnostic odds ratio of 71.7, 64.4, 68.4 , and $4.7 \%$, respectively. For SP-D, one study showed a sensitivity, specificity, accuracy, and diagnostic odds ratio of $70.0,65.0,68.5$, and $3.1 \%$, respectively. Translated into clinical practice, this implies that both MMP7 and SP-D serum levels indicate a correct IPF diagnosis in more than half of the patients while suggesting an incorrect classification in about one-third of patients. Based on these data, the diagnostic value of these serum biomarkers is currently considered insufficient to support clinical use.
Recommendation regarding the Use of Biomarkers to Diagnose IPF

In patients with newly diagnosed ILD of unknown cause and clinically suspected IPF, serum MMP7, SP-D, CCL18, or KL-6 levels shall not be measured for the purpose of IPF diagnosis and discrimination from other diseases.

\section{Diagnostic Criteria}

The required criteria for the diagnosis of IPF are criteria 1 and 2, or 1 and 3 :

1. Exclusion of known causes of ILD (exposure to inhaled noxious agents, CTD and other systemic diseases, drug-induced ILD, etc.) and either 2 or 3 ,

2. Presence of UIP pattern on HRCT (see Fig. 1; Table 2), 3. Specific HRCT/histology combinations (see Table 5).

If IPF is suspected, all patients are screened for possible ILD etiologies. In German-speaking countries, we recommend the use of a standardized Interstitial Lung Disease Patient Questionnaire developed by the clinical section of the DGP [68]. If a potential cause can be identified, it should be confirmed or excluded through further investigation. If the cause or diagnosis remains unclear despite a thorough evaluation, then the findings are to be discussed in the MDD meeting. IPF is diagnosed if the appropriate combination of HRCT and histopathological patterns is present (see Table 5). 
If an ILD is present and IPF is suspected (e.g., bilateral pulmonary infiltrates on chest X-ray or on CT, bibasilar inspiratory crackles, age $\geq 60$ years, unexplained exerciseinduced dyspnea, and/or cough), other causes of an ILD should be excluded first. This mainly relates to middleaged patients ( $>40$ and $<60$ years) and patients at risk for familial ILD. In such cases, other causes of ILD, for example, HP, CTD-ILD, pneumoconiosis, or medication use, should be thoroughly evaluated. This requires presenting the case in an initial MDD (Fig. 7). If no specific diagnosis is made, the clinical findings, the results of HRCT, and possibly bronchoalveolar lavage and/or cryobiopsy or SLB must be evaluated in a second MDD [150]. The diagnosis of IPF requires a specific combination of HRCT and (if available) histology (Table 5: combination of HRCT and histopathology).

Recommendations regarding the Diagnostic Process and BAL/Biopsy Procedures

1. For patients with suspected IPF and HRCT pattern of UIP without clinical signs of an alternative ILD diagnosis, no BAL and no biopsy shall be performed.

2. For patients with suspected IPF and HRCT pattern of UIP with clinical signs of an alternative ILD diagnosis (e.g., CTD-associated or chronic HP), BAL shall be performed.

3. For patients with suspected IPF and HRCT pattern of probable UIP, BAL but no biopsy shall be performed if the following criteria are met: $>60$ years of age, male, and ex-smoker. If the criteria are not met, a TBLC should be performed in addition.

4. For patients with suspected IPF who have an indeterminate for UIP or an alternative HRCT pattern, BAL and TBLC shall be performed.

5. For patients with suspected IPF who have undergone the endoscopic diagnostic procedures but where the diagnosis still remains unclear after the MDD, SLB shall be performed.

Annotation: all recommendations concerning cryobiopsy and SLB only apply if the patient-related safety criteria are fulfilled and if both the technical and personal expertise are available at the performing center.

\section{Scientific Questions and Outlook}

The expert panel sees an urgent need to further develop and validate the diagnostic procedures for ILDs. This concerns studies on the role of clinical parameters, HRCT, bronchoscopy, histopathology, and biomarkers.

\section{Clinical Parameters}

How should the disease behavior in individual patients be included in the diagnostic IPF algorithm? What is the role of patient-centered measurements like hand-held spirometry, accelerometry, or saturation measurements in this context? Could such patient-centered outcome measures, which in principle can be done at high frequency, possibly be even more sensitive than the periodic measurements of lung function and gas exchange at centers? Should systematic screening for comorbidities be part of the diagnostic evaluation to assess the prognosis? In patients with suspected IPF and probable UIP pattern on HRCT, to what extent does the observation of subsequent disease progression during the course of the disease contribute to validating the initial diagnosis of IPF and how does antifibrotic therapy influence this diagnostic implication?

A wait-and-watch approach for the natural course of the disease to confirm the diagnosis of IPF requires that the disease progresses in the majority of IPF patients within a certain time period. The inevitable consequence of such a strategy is that eligible patients do not receive antifibrotic therapy during this wait-and-watch period. The reliable identification of a "progressive chronic fibrosing" phenotype in patients based on one or clinical parameters or valid biomarkers is, therefore, of fundamental importance for IPF and beyond. Further studies will have to be conducted to answer these questions.

\section{HRCT}

What is the diagnostic significance of the extent and distribution pattern of traction bronchiectasis in patients with suspected IPF without visible honeycombing on HRCT? This also includes the question of the relative diagnostic value of central bronchiectasis and peripheral bronchiolectasis. Can the presence of mosaic attenuation, supported by a possibly obligatory expiratory CT scan, distinguish patients with chronic HP from IPF patients? How can mosaic attenuation be quantified, and can a standardized quantification contribute to differentiating the IPF-related UIP pattern from the UIP-like pattern of chronic HP? Can subgroups be formed based on the type and extent of GGO (by subjective assessment or automated methods) to estimate the probability of IPF? Does the craniocaudal distribution of fibrotic changes influence the probability of an IPF diagnosis? How is the HRCT interpretation influenced by the quality and quantity of available clinical information (concomitant diseases, inhaled noxious agents, etc.)? Can artificial intelligence be used to derive not only the correct diagnosis but 
also a "progressive chronic fibrosing" phenotype from a single HRCT? Can the interstitial lung abnormalities frequently observed in the early detection of lung cancer be further differentiated, based on radiological criteria, into those representing early stages of IPF and therefore requiring further diagnosis and therapy, and others that may potentially be nonprogressive and as such be insignificant in the further course of the disease?

\section{BAL and Transbronchial Lung Biopsy}

Will novel diagnostic procedures that use artificial intelligence and molecular signatures from brush swabs, lavages, or forceps biopsies or chemical signatures, for example, in expired air (electric nose) or exhaled breath condensate be suitable for a reliable differential diagnosis of IPF and for differentiating IPF from other ILDs? How often do BAL differential cell counts, histopathology results from transbronchial lung biopsies, and molecular profiling of transbronchial lung biopsies using a machine-learning algorithm $[121,122]$ provide additional, relevant information for the diagnosis of IPF? Can an internationally consented definition of the fibroblast focus and also of microscopic honeycombing be reached, followed by the standardized application of this definition to histopathological findings?

\section{Lung Cryobiopsy}

Experts familiar with the methods should - based on already published recommendations [143] - further develop an internationally accepted standard for lung cryobiopsy to improve the risk/benefit ratio and achieve the best possible diagnostic yield with the lowest possible complication rate and create a basis for international, multicenter studies. This may require further comparative studies and prospective register studies.

\section{Histopathology}

How often do the results of a SLB or cryobiopsy change the final diagnosis depending on the underlying HRCT pattern? Are there any relevant differences in this regard between surgical biopsy and cryobiopsy? What influence does SLB or cryobiopsy have on lung function parameters and clinical end points at different points in time after a biopsy?

\section{Genetic Markers and Counseling}

Is IPF a hereditary disease? Which genetic markers are present in patients in whom clinical manifestation indicates familial IPF or familial interstitial pneumonia, but none of the markers or gene mutations identified to date have been found despite molecular genetic studies? What is the relationship between mutations or abnormal genetic markers and intrinsic (e.g., microaspiration, lung microbiome, abnormal gastroesophageal reflux) or extrinsic/environmental factors? Should all IPF patients receive genetic counseling? Although genetic variants account for part of the risk of developing sporadic IPF or a familial form of ILD (i.e., familial IPF or familial interstitial pneumonia), the clinical benefit and clinical applicability of these variants are unclear and need to be determined in future studies.

\section{Other Biomarkers}

What is the optimal procedure to exclude ILD in CTD and chronic HP? What is the role of specific serum antibodies in excluding or confirming suspected chronic HP? Studies of diagnostic molecular biomarkers are needed to (a) assess the diagnostic accuracy of new potential biomarkers; (b) use machine learning to diagnose IPF; and (c) integrate molecular markers into the current multidisciplinary diagnostic process for IPF. New biomarkers for diagnosing IPF (molecular classifiers) could include exhaled breath markers (e.g., electric nose and exhaled breath condensate), circulating markers (serum/plasma proteins, cellular markers, epigenetic markers [miRNAs], mitochondrial DNA, etc.), as well as the ability to identify IPF or molecular signatures from lung samples (BAL, transbronchial biopsy, TBLC, lung microbiome), whereby the least invasive methods should be used to obtain samples. What is the added value of routine screening for germline mutations or polymorphisms or routine measurement of telomere length in suspected or confirmed IPF? Other unresolved issues beyond the scope of this guideline include the best procedure for assessing the prognosis, the identification of risk factors for developing IPF, the optimal strategy for early detection of IPF, and the procedure for recording concomitant diseases and determining their impact on the course of the disease in IPF patients.

\section{Summary of Recommendations}

\section{Recommendation regarding History of Exposure}

For patients with recently identified ILD of apparently unknown cause, who clinically meet the criteria of a presumptive IPF diagnosis, a detailed and complete history of both medication use and inhalational environmental exposure shall be obtained in a standardized format to exclude potential causes of ILD. 
Recommendation regarding Serological Tests

For patients with newly identified ILD of yet unknown cause who are clinically suspected of having IPF, serological testing shall generally be performed to identify a CTD as a potential cause of ILD.

\section{Diagnostic Imaging Recommendations}

Volumetric, high-resolution, non-contrast, inspiratory CT images shall be acquired for all patients with suspected IPF, with patients in supine position. Additional sequential HRCT slices in expiration should be acquired, if a disease of the small airways is suspected (e.g., bronchiolitis and HP). Findings can be supplemented with prone scans. An existing CT that does not meet the above quality criteria shall not be used for diagnostic purposes.

We recommend using the 4 HRCT diagnostic categories described in the publication of the Fleischner Society. These categories are "UIP pattern," "probable UIP pattern," "pattern indeterminate for UIP," and "alternative pattern." Radiology reports shall include an adequate description and apply the guideline-specific diagnostic criteria and classification system.

\section{Histopathology Recommendation}

The histopathology report shall include an adequate systematic description of alterations present and apply the diagnostic criteria and classification system of the guideline.

\section{Recommendations regarding $B A L$}

All patients with newly detected ILD of unknown cause and clinically suspected IPF shall undergo BAL if no definite UIP pattern is seen on HRCT.

If a definite UIP pattern on HRCT is present, BAL can be performed if there are concrete signs of an alternative etiology of the disease.

\section{Recommendation regarding Transbronchial Forceps Biopsy}

Transbronchial forceps biopsy shall not be performed for the diagnosis of IPF in patients with a definite UIP pattern on HRCT. No recommendation is provided for patients with other HRCT patterns.

\section{Recommendation regarding SLB}

For patients with suspected IPF who have undergone the endoscopic diagnostic procedures but where the diagnosis still remains unclear after the MDD, SLB shall be performed.

\section{Recommendations regarding TBLC}

In summary, the members of the German guideline panel - in contrast to the international guideline - considered TBLC to be preferable over surgical biopsy for the following reasons:

a. When compared to SLB, the diagnostic significance and side effect rates are not considered inferior.

b. The cost of TBLC versus SLB is considered to be lower, and it is expected that far fewer patients will refuse to undergo TBLC than surgery.

c. Patients with significantly advanced ILD can undergo biopsy using TBLC rather than SLB. This may also be the case for elderly patients and possibly for patients with multiple concomitant conditions. It is, therefore, expected that TBLC will reduce the percentage of unclassifiable ILDs.

The guideline panel, however, recommends that the following aspects of cryobiopsy, among others, be strictly observed [141]. On a critical note, it should be pointed out that the procedure has not yet been internationally standardized and that only little, partly contradictory data from controlled studies comparing TBLC versus SLB are available. Further research into this topic is encouraged.

\section{Recommendations regarding the Diagnostic Process and BAL/Biopsy Procedures}

1. For patients with suspected IPF and HRCT pattern of UIP without clinical signs of an alternative ILD diagnosis, no BAL and no biopsy shall be performed.

2. For patients with suspected IPF and HRCT pattern of UIP with clinical signs of an alternative ILD diagnosis (e.g., CTD-associated or chronic HP), BAL shall be performed.

3. For patients with suspected IPF and HRCT pattern of probable UIP, BAL but no biopsy shall be performed if the following criteria are met: $>60$ years of age, male, and ex-smoker. If the criteria are not met, a TBLC should be performed in addition.

4. For patients with suspected IPF who have an indeterminate for UIP or an alternative HRCT pattern, BAL and TBLC shall be performed.

5. For patients with suspected IPF who have undergone the endoscopic diagnostic procedures but where the diagnosis remains unclear after the MDD, SLB shall be performed.

Annotation: all recommendations concerning cryobiopsy and SLB only apply if the patient-related safety criteria are fulfilled and if both the technical and personal expertise are available at the performing center. 


\section{Recommendation regarding $M D D$}

The German guideline panel considers the MDD to be the diagnostic gold standard. An initial MDD conference shall be conducted with all available clinical information and HRCT findings to determine the further procedure.

After receiving the results of invasive diagnostic tests, the final diagnosis and further procedure shall then be determined in the context of a second MDD.

\section{Recommendation regarding the Use of Biomarkers for IPF Diagnosis}

In patients with newly diagnosed ILD of unknown cause and clinically suspected IPF, serum MMP7, SP-D, CCL18, or KL-6 levels shall not be measured for the purpose of IPF diagnosis and discrimination from other diseases.

\section{Acknowledgements}

The authors are indebted to Mrs. Gunda Mundt for expert translational service and to the German Respiratory Society (DGP e.V.) for funding of this work.

\section{Conflict of Interest Statement}

Jürgen Behr received honoraria for lectures and consultation from Actelion, Boehringer-Ingelheim, Roche, Biogen, BMS, Galapagos, Promedior, AstraZeneca, and Novartis. Andreas Günther received honoraria for lectures and consultation from BoehringerIngelheim and Roche. Francesco Bonella received honoraria for lectures and consultation from Boehringer-Ingelheim, Roche, Savara, Fujirebio, and Galapagos. Julien Dinkel received honoraria for lectures and consultation from Boehringer, MSD, and Beyen Consult. Ludger Fink received honoraria for lectures and consultation from Novartis and Roche. Thomas Geiser received honoraria for lectures and consultation from Boehringer-Ingelheim and Roche. Klaus Geißler has nothing to declare. Sven Gläser received honoraria for lectures and consultation from Actelion, AstraZeneca, Boehringer-Ingelheim, Berlin Chemie, Novartis, PneumRx, and Roche. Sabin Handzhhiev received honoraria for lectures and consultation from Boehringer-Ingelheim and Roche. Danny Jonigk received honoraria for lectures and consultation from Roche. Dirk Koschel received honoraria for lectures and consultation from Boehringer-Ingelheim and Roche. Michael Kreuter received honoraria for lectures and consultation from AstraZeneca, Bayer, Boehringer-Ingelheim, GlaxoSmithKline, Novartis, Chiesi, and Roche. Gabriela Leuschner received honoraria for lectures from AstraZeneca.Philipp Markart received honoraria for lectures and consultation from Berlin Chemie, Boehringer-Ingelheim, GSK, Novartis and Roche. Antje Prasse received honoraria for lectures and consultation from Boehringer-Ingelheim, Abbvie, Plinat Therapeutics, Roche, AstraZeneca and Novartis.Nicolas Schönfeld has nothing to declare. Jonas Christian Schupp received honoraria for lectures from Bayer AG. Helmut Sitter has nothing to declare. Joachim Müller-Quernheim received honoraria for lectures and consultation from AstraZeneca, Novartis, and Roche. Ulrich Costabel received honoraria for lectures and consultation from Boehringer-Ingelheim, Roche, Gobal Blood Therapeutics, and Fibrogen.

\section{References}

1 Behr J, Günther A, Ammenwerth W, Bittmann I, Bonnet R, Buhl R, et al. [German guideline for diagnosis and management of idiopathic pulmonary fibrosis]. Pneumologie. 2013;67(2):81-111.

2 Behr J, Günther A, Bonella F, Geissler K, Koschel D, Kreuter M, et al. [German Guideline for idiopathic pulmonary fibrosis: update on pharmacological therapies 2017]. Pneumologie. 2017;71(7):460-74.

3 Schunemann HJ, Jaeschke R, Cook DJ, Bria WF, El-Solh AA, Ernst A, et al. An official ATS statement: grading the quality of evidence and strength of recommendations in ATS guidelines and recommendations. Am J Respir Crit Care Med. 2006;174(5):605-14.

4 Travis WD, Costabel U, Hansell DM, King TE Jr, Lynch DA, Nicholson AG, et al. An official American Thoracic Society/European Respiratory Society statement: update of the international multidisciplinary classification of the idiopathic interstitial pneumonias. Am J Respir Crit Care Med. 2013;188(6):733-48.
5 Raghu G, Remy-Jardin M, Myers JL, Richeldi L, Ryerson CJ, Lederer DJ, et al. Diagnosis of idiopathic pulmonary fibrosis. An Official ATS/ERS/JRS/ALAT clinical practice guideline. Am J Respir Crit Care Med. 2018;198(5): e44-68.

6 Wells AU, Brown KK, Flaherty KR, Kolb M, Thannickal VJ; IPF Consensus Working Group. What's in a name? That which we call IPF, by any other name would act the same. Eur Respir J. 2018;51(5):1800692.

7 Olson AL, Gifford AH, Inase N, Fernández Pérez ER, Suda T. The epidemiology of idiopathic pulmonary fibrosis and interstitial lung diseases at risk of a progressive-fibrosing phenotype. Eur Respir Rev. 2018;27(150): 180077.

8 Duchemann B, Annesi-Maesano I, Jacobe de Naurois C, Sanyal S, Brillet PY, Brauner M, et al. Prevalence and incidence of interstitial lung diseases in a multi-ethnic county of Greater Paris. Eur Respir J. 2017;50(2):1602419.
9 Wuyts WA, Dahlqvist C, Slabbynck H, Schlesser M, Gusbin N, Compere C, et al. Baseline clinical characteristics, comorbidities and prescribed medication in a real-world population of patients with idiopathic pulmonary fibrosis: the PROOF registry. BMJ Open Respir Res. 2018;5(1):e000331.

10 Collard HR, Tino G, Noble PW, Shreve MA, Michaels M, Carlson B, et al. Patient experiences with pulmonary fibrosis. Respir Med. 2007;101(6):1350-4.

11 Richeldi L, du Bois RM, Raghu G, Azuma A, Brown KK, Costabel U, et al. Efficacy and safety of nintedanib in idiopathic pulmonary fibrosis. N Engl J Med. 2014;370(22):2071-82.

12 Noble PW, Albera C, Bradford WZ, Costabel U, du Bois RM, Fagan EA, et al. Pirfenidone for idiopathic pulmonary fibrosis: analysis of pooled data from three multinational phase 3 trials. Eur Respir J. 2016;47(1):243-53.

13 King TE Jr, Bradford WZ, Castro-Bernardini S, Fagan EA, Glaspole I, Glassberg MK, et al. A phase 3 trial of pirfenidone in patients with idiopathic pulmonary fibrosis. N Engl J Med. 2014;370(22):2083-92. 
14 Behr J, Kreuter M, Hoeper MM, Wirtz H, Klotsche J, Koschel D, et al. Management of patients with idiopathic pulmonary fibrosis in clinical practice: the INSIGHTS-IPF registry. Eur Respir J. 2015;46(1):186-96.

15 Ferrara G, Carlson L, Palm A, Einarsson J, Olivesten C, Skold M. Idiopathic pulmonary fibrosis in Sweden: report from the first year of activity of the Swedish IPF-Registry. Eur Clin Respir J. 2016;3:31090.

16 Jo HE, Glaspole I, Grainge C, Goh N, Hopkins PM, Moodley Y, et al. Baseline characteristics of idiopathic pulmonary fibrosis: analysis from the Australian Idiopathic Pulmonary Fibrosis Registry. Eur Respir J. 2017;49(2): 1601592.

17 Guenther A, Krauss E, Tello S, Wagner J, Paul B, Kuhn S, et al. The European IPF registry (eurIPFreg): baseline characteristics and survival of patients with idiopathic pulmonary fibrosis. Respir Res. 2018;19(1):141

18 Doubkova M, Svancara J, Svoboda M, Sterclova M, Bartos V, Plackova M, et al. EMPIRE Registry, Czech Part: impact of demographics, pulmonary function and HRCT on survival and clinical course in idiopathic pulmonary fibrosis. Clin Respir J. 2018;12(4):152635.

19 Torrisi SE, Ley B, Kreuter M, Wijsenbeek M, Vittinghoff E, Collard HR, et al. The added value of comorbidities in predicting survival in idiopathic pulmonary fibrosis: a multicentre observational study. Eur Respir J. 2019; 53(3): 1801587

20 Nadrous HF, Myers JL, Decker PA, Ryu JH. Idiopathic pulmonary fibrosis in patients younger than 50 years. Mayo Clin Proc. 2005 80(1):37-40.

21 Ley B, Collard HR, King TE Jr. Clinical course and prediction of survival in idiopathic pulmonary fibrosis. Am J Respir Crit Care Med. 2011;183(4):431-40.

22 Kolb M, Richeldi L, Behr J, Maher TM, Tang W, Stowasser S, et al. Nintedanib in patients with idiopathic pulmonary fibrosis and preserved lung volume. Thorax. 2017;72(4):340-

23 Costabel U, Crestani B, Wells A. Idiopathic pulmonary fibrosis. ERS Monograph. 2016; 71

24 Kreuter M, Ehlers-Tenenbaum S, Palmowsk $\mathrm{K}$, Bruhwyler J, Oltmanns U, Muley T, et al. Impact of comorbidities on mortality in patients with idiopathic pulmonary fibrosis PLoS One. 2016;11(3):e0151425.

25 Raghu G, Amatto VC, Behr J, Stowasser S. Comorbidities in idiopathic pulmonary fibrosis patients: a systematic literature review. Eur Respir J. 2015;46(4):1113-30.

26 Costabel U, Behr J, Crestani B, Stansen W, Schlenker-Herceg R, Stowasser S, et al. Antiacid therapy in idiopathic pulmonary fibrosis: insights from the INPULSIS(R) trials. Respir Res. 2018;19(1):167.
27 Kato E, Takayanagi N, Takaku Y, Kagiyama $\mathrm{N}$, Kanauchi T, Ishiguro $\mathrm{T}$, et al. Incidence and predictive factors of lung cancer in patients with idiopathic pulmonary fibrosis. ERJ Open Res. 2018;4(1).

28 Ozawa Y, Suda T, Naito T, Enomoto N Hashimoto D, Fujisawa T, et al. Cumulative incidence of and predictive factors for lung cancer in IPF. Respirology. 2009;14(5):723-8.

29 Tomassetti S, Gurioli C, Ryu JH, Decker PA, Ravaglia C, Tantalocco P, et al. The impact of lung cancer on survival of idiopathic pulmonary fibrosis. Chest. 2015;147(1):157-64.

30 Song JW, Hong SB, Lim CM, Koh Y, Kim DS Acute exacerbation of idiopathic pulmonary fibrosis: incidence, risk factors and outcome. Eur Respir J. 2011;37(2):356-63.

31 Collard HR, Ryerson CJ, Corte TJ, Jenkins G, Kondoh Y, Lederer DJ, et al. Acute exacerbation of idiopathic pulmonary fibrosis. an International Working Group Report. Am J Respir Crit Care Med. 2016;194(3):265-75.

32 Lynch DA, Sverzellati N, Travis WD, Brown KK, Colby TV, Galvin JR, et al. Diagnostic criteria for idiopathic pulmonary fibrosis: a Fleischner Society White Paper. Lancet Respir Med. 2018;6(2):138-53.

33 Kubo T, Lin PJ, Stiller W, Takahashi M, Kauczor HU, Ohno Y, et al. Radiation dose reduction in chest CT: a review. AJR Am J Roentgenol. 2008;190(2):335-43.

34 Remy-Jardin M, Campistron P, Amara A, Mastora I, Tillie-Leblond I, Delannoy V, et al. Usefulness of coronal reformations in the diagnostic evaluation of infiltrative lung disease. J Comput Assist Tomogr. 2003;27(2): 266-73.

35 Mayo JR. CT evaluation of diffuse infiltrative lung disease: dose considerations and optimal technique. J Thorac Imaging. 2009;24(4): 252-9.

36 Nagel H, Hering K, Hieckel H, Ludwig K, Rehbock B, Hofmann-Preiß K. Protokollempfehlungen der AG DRauE zur Durchführung von Low-Dose-Volumen-HRCT-Untersuchungen der Lunge. Fortschr Röntengstr. 2017; 189:553-75.

37 Miller WT Jr, Chatzkel J, Hewitt MG. Expiratory air trapping on thoracic computed tomography. A diagnostic subclassification. Ann Am Thorac Soc. 2014;11(6):874-81.

38 Kim M, Lee SM, Song JW, Do KH, Lee HJ, Lim S, et al. Added value of prone CT in the assessment of honeycombing and classification of usual interstitial pneumonia pattern. Eur J Radiol. 2017;91:66-70.

39 Bankier AA, O’Donnell CR, Boiselle PM. Quality initiatives. Respiratory instructions for CT examinations of the lungs: a hands-on guide. Radiographics. 2008;28(4):919-31.

40 Hansell DM, Bankier AA, MacMahon $\mathrm{H}$, McLoud TC, Müller NL, Remy J. Fleischner Society: glossary of terms for thoracic imaging. Radiology. 2008;246(3):697-722.
41 Wormanns D, Hamer O. Glossar thoraxradiologischer Begriffe entsprechend der Terminologie der Fleischner Society. Fortschr Röntgenstr. 2015;187(8):638-61.

42 Watadani T, Sakai F, Johkoh T, Noma S, Akira M, Fujimoto K, et al. Interobserver variability in the CT assessment of honeycombing in the lungs. Radiology. 2013;266(3):936-44.

43 Lynch DA, Godwin JD, Safrin S, Starko KM, Hormel P, Brown KK, et al. High-resolution computed tomography in idiopathic pulmonary fibrosis: diagnosis and prognosis. Am J Respir Crit Care Med. 2005;172(4):488-93.

44 Walsh SL, Sverzellati N, Devaraj A, Wells AU, Hansell DM. Chronic hypersensitivity pneumonitis: high resolution computed tomography patterns and pulmonary function indices as prognostic determinants. Eur Radiol. 2012; 22(8):1672-9.

45 Edey AJ, Devaraj AA, Barker RP, Nicholson AG, Wells AU, Hansell DM. Fibrotic idiopathic interstitial pneumonias: HRCT findings that predict mortality. Eur Radiol. 2011; 21(8):1586-93.

46 Remy-Jardin M, Giraud F, Remy J, Copin MC, Gosselin B, Duhamel A. Importance of ground-glass attenuation in chronic diffuse infiltrative lung disease: pathologic-CT correlation. Radiology. 1993;189(3):693-8.

47 Akira M, Kozuka T, Yamamoto S, Sakatani M Computed tomography findings in acute exacerbation of idiopathic pulmonary fibrosis. Am J Respir Crit Care Med. 2008;178(4):372-8.

48 Collard HR, Moore BB, Flaherty KR, Brown KK, Kaner RJ, King TE Jr, et al. Acute exacerbations of idiopathic pulmonary fibrosis. Am J Respir Crit Care Med. 2007;176(7):636-43.

49 Gruden JF, Panse PM, Leslie KO, Tazelaar HD, Colby TV. UIP diagnosed at surgical lung biopsy, 2000-2009: HRCT patterns and proposed classification system. AJR Am J Roentgenol. 2013;200(5):W458-67.

50 Tcherakian C, Cottin V, Brillet PY, Freynet O, Naggara N, Carton Z, et al. Progression of idiopathic pulmonary fibrosis: lessons from asymmetrical disease. Thorax. 2011;66(3): $226-31$.

51 Hunninghake GW, Zimmerman MB, Schwartz DA, King TE Jr, Lynch J, Hegele R, et al. Utility of a lung biopsy for the diagnosis of idiopathic pulmonary fibrosis. Am J Respir Crit Care Med. 2001;164(2):193-6.

52 Swensen SJ, Aughenbaugh GL, Myers JL. Diffuse lung disease: diagnostic accuracy of CT in patients undergoing surgical biopsy of the lung. Radiology. 1997;205(1):229-34.

53 Raghu G, Mageto YN, Lockhart D, Schmidt RA, Wood DE, Godwin JD. The accuracy of the clinical diagnosis of new-onset idiopathic pulmonary fibrosis and other interstitial lung disease: a prospective study. Chest. 1999; 116(5):1168-74.

54 Souza CA, Müller NL, Lee KS, Johkoh T, Mitsuhiro $\mathrm{H}$, Chong $\mathrm{S}$. Idiopathic interstitial pneumonias: prevalence of mediastinal lymph node enlargement in 206 patients. AJR Am J Roentgenol. 2006;186(4):995-9. 
55 Egashira R, Jacob J, Kokosi MA, Brun AL, Rice A, Nicholson AG, et al. Diffuse pulmonary ossification in fibrosing interstitial lung diseases: prevalence and associations. Radiology. 2017;284(1):255-63.

56 Reddy TL, von der Thüsen J, Walsh SL. Idiopathic dendriform pulmonary ossification. J Thorac Imaging. 2012;27(5):W108-10.

57 Reddy TL, Tominaga M, Hansell DM, von der Thusen J, Rassl D, Parfrey H, et al. Pleuroparenchymal fibroelastosis: a spectrum of histopathological and imaging phenotypes. Eur Respir J. 2012;40(2):377-85.

58 Inomata M, Ikushima S, Awano N, Kondoh K, Satake K, Masuo M, et al. An autopsy study of combined pulmonary fibrosis and emphysema: correlations among clinical, radiological, and pathological features. BMC Pulm Med. 2014;14:104.

59 Chung JH, Chawla A, Peljto AL, Cool CD, Groshong SD, Talbert JL, et al. CT scan findings of probable usual interstitial pneumonitis have a high predictive value for histologic usual interstitial pneumonitis. Chest. 2015; 147(2):450-9.

60 Salisbury ML, Xia M, Murray S, Bartholmai BJ, Kazerooni EA, Meldrum CA, et al. Predictors of idiopathic pulmonary fibrosis in absence of radiologic honeycombing: a cross sectional analysis in ILD patients undergoing lung tissue sampling. Respir Med. 2016;118: 88-95.

61 Yagihashi K, Huckleberry J, Colby TV, Tazelaar HD, Zach J, Sundaram B, et al. Radiologic-pathologic discordance in biopsy-proven usual interstitial pneumonia. Eur Respir J. 2016;47(4):1189-97.

62 Raghu G, Collard HR, Egan JJ, Martinez FJ, Behr J, Brown KK, et al. An official ATS/ERS/ JRS/ALAT statement: idiopathic pulmonary fibrosis: evidence-based guidelines for diagnosis and management. Am J Respir Crit Care Med. 2011;183(6):788-824.

63 Langer F, Stark H, Braubach P, Ackermann $\mathrm{M}$, Hussein $\mathrm{K}$, Teiken K, et al. [Injury patterns in interstitial lung diseases]. Pathologe. 2018; 39(Suppl 2):262-71.

64 Fink L. [Interstitial lung diseases. The pattern is important]. Pathologe. 2014;35(6):597605.

65 Singh S, Collins BF, Sharma BB, Joshi JM, Talwar D, Katiyar S, et al. Interstitial lung disease in India. Results of a prospective registry. Am J Respir Crit Care Med. 2017;195(6):801-13.

66 Salisbury ML, Myers JL, Belloli EA, Kazerooni EA, Martinez FJ, Flaherty KR. Diagnosis and treatment of fibrotic hypersensitivity pneumonia. Where we stand and where we need to go. Am J Respir Crit Care Med. 2017;196(6): $690-9$.

67 Vasakova M, Morell F, Walsh S, Leslie K, Raghu G. Hypersensitivity pneumonitis: perspectives in diagnosis and management. Am J Respir Crit Care Med. 2017;196(6): $680-9$.
68 Kreuter M, Ochmann U, Koschel D, Behr J, Bonella F, Claussen M, et al. Patientenfragebogen zur Erfassung der Ursachen interstitieller und seltener Lungenerkrankungen: klinische Sektion der DGP. Pneumologie. 2018;72(6):446-57.

69 Miyake Y, Sasaki S, Yokoyama T, Chida K, Azuma A, Suda T, et al. Occupational and environmental factors and idiopathic pulmonary fibrosis in Japan. Ann Occup Hyg. 2005; 49(3):259-65.

70 Iwai K, Mori T, Yamada N, Yamaguchi M, Hosoda Y. Idiopathic pulmonary fibrosis. Epidemiologic approaches to occupational exposure. Am J Respir Crit Care Med. 1994; 150(3):670-5

71 Hubbard R, Cooper M, Antoniak M, Venn A, Khan S, Johnston I, et al. Risk of cryptogenic fibrosing alveolitis in metal workers. Lancet. 2000;355(9202):466-7.

72 Baumgartner KB, Samet JM, Stidley CA, Colby TV, Waldron JA. Cigarette smoking: a risk factor for idiopathic pulmonary fibrosis. Am J Respir Crit Care Med. 1997;155(1):242-8.

73 Johnston ID, Prescott RJ, Chalmers JC, Rudd RM. British Thoracic Society study of cryptogenic fibrosing alveolitis: current presentation and initial management. Fibrosing $\mathrm{Al}$ veolitis Subcommittee of the Research Committee of the British Thoracic Society. Thorax. 1997;52(1):38-44.

74 Hubbard R, Lewis S, Richards K, Johnston I, Britton J. Occupational exposure to metal or wood dust and aetiology of cryptogenic fibrosing alveolitis. Lancet. 1996;347(8997): 284-9.

75 Gustafson T, Dahlman-Höglund A, Nilsson K, Ström K, Tornling G, Torén K. Occupational exposure and severe pulmonary fibrosis. Respir Med. 2007;101(10):2207-12.

76 Fischer A, Antoniou KM, Brown KK, Cadranel J, Corte TJ, du Bois RM, et al. An official European Respiratory Society/American Thoracic Society research statement: interstitial pneumonia with autoimmune features. Eur Respir J. 2015;46(4):976-87.

77 Lega JC, Reynaud Q, Belot A, Fabien N, Durieu I, Cottin V. Idiopathic inflammatory myopathies and the lung. Eur Respir Rev. 2015; 24(136):216-38.

78 Lee W, Chung WS, Hong KS, Huh J. Clinical usefulness of bronchoalveolar lavage cellular analysis and lymphocyte subsets in diffuse interstitial lung diseases. Ann Lab Med. 2015; 35(2):220-5.

79 Schildge J, Frank J, Klar B. [The role of bronchoalveolar lavage in the diagnosis of idiopathic pulmonary fibrosis: an investigation of the relevance of the protein content]. Pneumologie. 2016;70(7):435-41.

80 Nagai S, Kitaichi M, Itoh H, Nishimura K, Izumi T, Colby TV. Idiopathic nonspecific interstitial pneumonia/fibrosis: comparison with idiopathic pulmonary fibrosis and BOOP. Eur Respir J. 1998;12(5):1010-9.
81 Ohshimo S, Bonella F, Cui A, Beume M, Kohno N, Guzman J, et al. Significance of bronchoalveolar lavage for the diagnosis of idiopathic pulmonary fibrosis. Am J Respir Crit Care Med. 2009;179(11):1043-7.

82 Efared B, Ebang-Atsame G, Rabiou S, Diarra AS, Tahiri L, Hammas N, et al. The diagnostic value of the bronchoalveolar lavage in interstitial lung diseases. J Negat Results Biomed. 2017;16(1):4

83 Welker L, Jörres RA, Costabel U, Magnussen $H$. Predictive value of BAL cell differentials in the diagnosis of interstitial lung diseases. Eur Respir J. 2004;24(6):1000-6.

84 Ryu YJ, Chung MP, Han J, Kim TS, Lee KS, Chun EM, et al. Bronchoalveolar lavage in fibrotic idiopathic interstitial pneumonias. Respir Med. 2007;101(3):655-60.

85 Veeraraghavan S, Latsi PI, Wells AU, Pantelidis P, Nicholson AG, Colby TV, et al. BAL findings in idiopathic nonspecific interstitial pneumonia and usual interstitial pneumonia. Eur Respir J. 2003;22(2):239-44.

86 Schildge J, Nagel C, Grun C. Bronchoalveolar lavage in interstitial lung diseases: does the recovery rate affect the results? Respiration. 2007;74(5):553-7.

87 Ayed AK. Video-assisted thoracoscopic lung biopsy in the diagnosis of diffuse interstitial lung disease. A prospective study. J Cardiovasc Surg. 2003;44(1):115-8.

88 Morris D, Zamvar V. The efficacy of videoassisted thoracoscopic surgery lung biopsies in patients with interstitial lung disease: a retrospective study of 66 patients. J Cardiothorac Surg. 2014;9:45.

89 Bagheri R, Haghi SZ, Attaran D, Hashem Asnaashari AM, Basiri R, Rajabnejad A. Efficacy of minimally invasive surgery in diagnosis of interstitial lung disease. Asian Cardiovasc Thorac Ann. 2015;23(7):851-4.

90 Bando M, Ohno S, Hosono T, Yanase K, Sato $\mathrm{Y}$, Sohara Y, et al. Risk of acute exacerbation after video-assisted thoracoscopic lung biopsy for interstitial lung disease. J Bronchology Interv Pulmonol. 2009;16(4):229-35.

91 Blackhall V, Asif M, Renieri A, Civitelli S, Kirk A, Jilaihawi A, et al. The role of surgical lung biopsy in the management of interstitial lung disease: experience from a single institution in the UK. Interact Cardiovasc Thorac Surg. 2013;17(2):253-7.

92 Blanco M, Obeso GA, Durán JC, Rivo JE, García-Fontán E, Peña E, et al. Surgical lung biopsy for diffuse lung disease. Our experience in the last 15 years. Rev Port Pneumol. 2013; 19(2):59-64.

93 Blewett CJ, Bennett WF, Miller JD, Urschel JD. Open lung biopsy as an outpatient procedure. Ann Thorac Surg. 2001;71(4):1113-5.

94 Fibla JJ, Brunelli A, Allen MS, Wigle D, Shen $\mathrm{R}$, Nichols F, et al. Do the number and volume of surgical lung biopsies influence the diagnostic yield in interstitial lung disease? A propensity score analysis. Arch Bronconeumol. 2015;51(2):76-9. 
95 Guerra M, Miranda JA, Leal F, Vouga L. Interstitial lung disease: diagnostic accuracy and safety of surgical lung biopsy. Rev Port Pneumol. 2009;15(3):433-42.

96 Ishie RT, Cardoso JJ, Silveira RJ, Stocco L. Video-assisted thoracoscopy for the diagnosis of diffuse parenchymal lung disease. J Bras Pneumol. 2009;35(3):234-41.

97 Kayatta MO, Ahmed S, Hammel JA, Fernandez F, Pickens A, Miller D, et al. Surgical biopsy of suspected interstitial lung disease is superior to radiographic diagnosis. Ann Thorac Surg. 2013;96(2):399-401.

98 Khalil M, Cowen M, Chaudhry M, Loubani M. Single versus multiple lung biopsies for suspected interstitial lung disease. Asian Cardiovasc Thorac Ann. 2016;24(8):788-91.

99 Kreider ME, Hansen-Flaschen J, Ahmad NN, Rossman MD, Kaiser LR, Kucharczuk JC, et al. Complications of video-assisted thoracoscopic lung biopsy in patients with interstitial lung disease. Ann Thorac Surg. 2007;83(3):1140-4.

100 Luo Q, Han Q, Chen X, Xie J, Wu L, Chen R. The diagnosis efficacy and safety of videoassisted thoracoscopy surgery (VATS) in undefined interstitial lung diseases: a retrospective study. J Thorac Dis. 2013;5(3):2838.

101 Miller JD, Urschel JD, Cox G, Olak J, Young JE, Kay JM, et al. A randomized, controlled trial comparing thoracoscopy and limited thoracotomy for lung biopsy in interstitial lung disease. Ann Thorac Surg. 2000;70(5): 1647-50.

102 Ooi A, Iyenger S, Ferguson J, Ritchie AJ. VATS lung biopsy in suspected, diffuse interstitial lung disease provides diagnosis, and alters management strategies. Heart Lung Circ. 2005;14(2):90-2.

103 Pompeo E, Rogliani P, Cristino B, Schillaci O, Novelli G, Saltini C. Awake thoracoscopic biopsy of interstitial lung disease. Ann Thorac Surg. 2013;95(2):445-52.

104 Qureshi RA, Ahmed TA, Grayson AD, Soorae AS, Drakeley MJ, Page RD. Does lung biopsy help patients with interstitial lung disease? Eur J Cardiothorac Surg. 2002; 21(4):621-6; .

105 Rotolo N, Imperatori A, Dominioni L, Facchini A, Conti V, Castiglioni M, et al. Efficacy and safety of surgical lung biopsy for interstitial disease. Experience of 161 consecutive patients from a single institution in Italy. Sarcoidosis Vasc Diffuse Lung Dis. 2015;32(3):251-8.

106 Samejima J, Tajiri M, Ogura T, Baba T, Omori T, Tsuboi M, et al. Thoracoscopic lung biopsy in 285 patients with diffuse pulmonary disease. Asian Cardiovasc Thorac Ann. 2015;23(2):191-7.

107 Sigurdsson MI, Isaksson HJ, Gudmundsson G, Gudbjartsson T. Diagnostic surgical lung biopsies for suspected interstitial lung diseases: a retrospective study. Ann Thorac Surg. 2009;88(1):227-32.
108 Sonobe M, Handa T, Tanizawa K, Sato M, Sato T, Chen F, et al. Videothoracoscopyassisted surgical lung biopsy for interstitial lung diseases. Gen Thorac Cardiovasc Surg. 2014;62(6):376-82.

109 Tomassetti S, Wells AU, Costabel U, Cavazza A, Colby TV, Rossi G, et al. Bronchoscopic lung cryobiopsy increases diagnostic confidence in the multidisciplinary diagnosis of idiopathic pulmonary fibrosis. Am J Respir Crit Care Med. 2016;193(7):745-52.

110 Ravaglia C, Bonifazi M, Wells AU, Tomassetti S, Gurioli C, Piciucchi S, et al. Safety and diagnostic yield of transbronchial lung cryobiopsy in diffuse parenchymal lung diseases: a comparative study versus video-assisted thoracoscopic lung biopsy and a systematic review of the literature. Respiration. 2016; 91(3):215-27.

111 Morell F, Reyes L, Doménech G, De Gracia J, Majó J, Ferrer J. [Diagnoses and diagnostic procedures in 500 consecutive patients with clinical suspicion of interstitial lung disease]. Arch Bronconeumol. 2008;44(4):18591.

112 Findikcioglu A, Kilic D, Karadayi S, Canpolat $\mathrm{D}$, Hatipoglu A. Is surgical biopsy necessary for diagnosis of interstitial lung disease: a retrospective clinical study. J Clin Anal Med. 2014;5:204-8.

113 Fisher JH, Shapera S, To T, Marras TK, Gershon A, Dell S. Procedure volume and mortality after surgical lung biopsy in interstitial lung disease. Eur Respir J. 2019;53(2): 1801164.

114 Han Q, Luo Q, Chen X, Xie J, Wu L, Chen R. The evaluation of clinical usefulness of transbrochoscopic lung biopsy in undefined interstitial lung diseases: a retrospective study. Clin Respir J. 2017;11(2):168-75.

115 Sindhwani G, Shirazi N, Sodhi R, Raghuvanshi S, Rawat J. Transbronchial lung biopsy in patients with diffuse parenchymal lung disease without 'idiopathic pulmonary fibrosis pattern' on HRCT scan: experience from a tertiary care center of North India. Lung India. 2015;32(5):453-6.

116 Sheth JS, Belperio JA, Fishbein MC, Kazerooni EA, Lagstein A, Murray S, et al. Utility of transbronchial vs surgical lung biopsy in the diagnosis of suspected fibrotic interstitial lung disease. Chest. 2017;151(2):389-99.

117 Pajares V, Puzo C, Castillo D, Lerma E, Montero MA, Ramos-Barbón D, et al. Diagnostic yield of transbronchial cryobiopsy in interstitial lung disease: a randomized trial. Respirology. 2014;19(6):900-6.

118 Pourabdollah M, Shamaei M, Karimi S, Karimi M, Kiani A, Jabbari HR. Transbronchial lung biopsy: the pathologist's point of view. Clin Respir J. 2016;10(2):211-6.

119 Ramaswamy A, Homer R, Killam J, Pisani MA, Murphy TE, Araujo K, et al. Comparison of transbronchial and cryobiopsies in evaluation of diffuse parenchymal lung disease. J Bronchology Interv Pulmonol. 2016; 23(1):14-21.
120 Wall CP, Gaensler EA, Carrington CB, Hayes JA. Comparison of transbronchial and open biopsies in chronic infiltrative lung diseases. Am Rev Respir Dis. 1981; 123(3):280-5.

121 Kim SY, Diggans J, Pankratz D, Huang J, Pagan M, Sindy N, et al. Classification of usual interstitial pneumonia in patients with interstitial lung disease: assessment of a machine learning approach using high-dimensional transcriptional data. Lancet Respir Med. 2015;3(6):473-82.

122 Pankratz DG, Choi Y, Imtiaz U, Fedorowicz GM, Anderson JD, Colby TV, et al. Usual interstitial pneumonia can be detected in transbronchial biopsies using machine learning. Ann Am Thorac Soc. 2017;14(11):1646-54.

123 Cascante JA, Cebollero P, Herrero S, Yagüe A, Echegoyen A, Elizalde J, et al. Transbronchial cryobiopsy in interstitial lung disease: are we on the right path? J Bronchology Interv Pulmonol. 2016;23(3):204-9.

124 Fruchter O, Fridel L, El Raouf BA, AbdelRahman N, Rosengarten D, Kramer MR. Histological diagnosis of interstitial lung diseases by cryo-transbronchial biopsy. Respirology. 2014;19(5):683-8.

125 Griff S, Schönfeld N, Ammenwerth W, Blum TG, Grah C, Bauer TT, et al. Diagnostic yield of transbronchial cryobiopsy in non-neoplastic lung disease: a retrospective case series. BMC Pulm Med. 2014;14:171.

126 Hagmeyer L, Theegarten D, Treml M, Priegnitz C, Randerath W. Validation of transbronchial cryobiopsy in interstitial lung disease: interim analysis of a prospective trial and critical review of the literature. Sarcoidosis Vasc Diffuse Lung Dis. 2016;33(1):2-9.

127 Hernandez-Gonzalez F, Lucena CM, Ramirez J, Sanchez M, Jimenez MJ, Xaubet A, et al. Cryobiopsy in the diagnosis of diffuse interstitial lung disease: yield and costeffectiveness analysis. Arch Bronconeumol. 2015;51(6):261-7.

128 Kronborg-White S, Folkersen B, Rasmussen TR, Voldby N, Madsen LB, Rasmussen F, et al. Introduction of cryobiopsies in the diagnostics of interstitial lung diseases: experiences in a referral center. Eur Clin Respir J. 2017;4(1):1274099.

129 Kropski JA, Pritchett JM, Mason WR, Sivarajan L, Gleaves LA, Johnson JE, et al. Bronchoscopic cryobiopsy for the diagnosis of diffuse parenchymal lung disease. PLoS One. 2013;8(11):e78674.

130 Ussavarungsi K, Kern RM, Roden AC, Ryu JH, Edell ES. Transbronchial cryobiopsy in diffuse parenchymal lung disease: retrospective analysis of 74 cases. Chest. 2017;151(2): $400-8$.

131 Hetzel J, Eberhardt R, Petermann C, Gesierich W, Darwiche K, Hagmeyer L, et al. Bleeding risk of transbronchial cryobiopsy compared to transbronchial forceps biopsy in interstitial lung disease: a prospective, randomized, multicentre cross-over trial. Respir Res. 2019;20(1):140. 
132 Hagmeyer L, Theegarten D, Wohlschläger J, Hager T, Treml M, Herkenrath SD, et al. Transbronchial cryobiopsy in fibrosing interstitial lung disease: modifications of the procedure lead to risk reduction. Thorax. 2019;74(7):711-4

133 Romagnoli M, Colby TV, Berthet JP, Gamez AS, Mallet JP, Serre I, et al. Poor Concordance between sequential transbronchial lung cryobiopsy and surgical lung biopsy in the diagnosis of diffuse interstitial lung diseases. Am J Respir Crit Care Med. 2019; 199(10):1249-56.

134 Troy LK, Grainge C, Corte TJ, Williamson JP, Vallely MP, Cooper WA, et al. Diagnostic accuracy of transbronchial lung cryobiopsy for interstitial lung disease diagnosis (COLDICE): a prospective, comparative study. Lancet Respir Med. 2020 Feb;8(2):171-81.

135 Ravaglia C, Wells AU, Tomassetti S, Gurioli C, Gurioli C, Dubini A, et al. Diagnostic yield and risk/benefit analysis of trans-bronchial lung cryobiopsy in diffuse parenchymal lung diseases: a large cohort of 699 patients. BMC Pulm Med. 2019;19(1):16.

136 Lentz RJ, Argento AC, Colby TV, Rickman OB, Maldonado F. Transbronchial cryobiopsy for diffuse parenchymal lung disease: a state-of-the-art review of procedural techniques, current evidence, and future challenges. J Thorac Dis. 2017;9(7):2186-203.

137 Walscher J, Gross B, Eberhardt R, Heussel $\mathrm{CP}$, Eichinger M, Warth A, et al. Transbronchial cryobiopsies for diagnosing interstitial lung disease: real-life experience from a Tertiary Referral Center for interstitial lung disease. Respiration. 2019;97(4):348-54.

138 Lentz RJ, Taylor TM, Kropski JA, Sandler KL, Johnson JE, Blackwell TS, et al. Utility of flexible bronchoscopic cryobiopsy for diagnosis of diffuse parenchymal lung diseases. J Bronchology Interv Pulmonol. 2018;25(2): 88-96.
139 Cooley J, Balestra R, Aragaki-Nakahodo AA, Caudell Stamper DN, Sriprasart T, Swank Z, et al. Safety of performing transbronchial lung cryobiopsy on hospitalized patients with interstitial lung disease. Respir Med. 2018;140:71-6.

140 Dhooria S, Mehta RM, Srinivasan A, Madan K, Sehgal IS, Pattabhiraman V, et al. The safety and efficacy of different methods for obtaining transbronchial lung cryobiopsy in diffuse lung diseases. Clin Respir J. 2018; 12(4):1711-20.

141 Bondue B, Pieters T, Alexander P, De Vuyst P, Ruiz Patino M, Hoton D, et al. Role of transbronchial lung cryobiopsies in diffuse parenchymal lung diseases: interest of a sequential approach. Pulm Med. 2017;2017: 6794343.

142 Sharp C, McCabe M, Adamali H, Medford AR. Use of transbronchial cryobiopsy in the diagnosis of interstitial lung disease-a systematic review and cost analysis. QJM. 2017; 110(4):207-14.

143 Hetzel J, Maldonado F, Ravaglia C, Wells AU, Colby TV, Tomassetti S, et al. Transbronchial cryobiopsies for the diagnosis of diffuse parenchymal lung diseases: expert statement from the Cryobiopsy Working Group on safety and utility and a call for standardization of the procedure. Respiration. 2018;95(3):188-200.

144 Chaudhuri N, Spencer L, Greaves M, Bishop P, Chaturvedi A, Leonard C. A review of the multidisciplinary diagnosis of interstitial lung diseases: a retrospective analysis in a single UK Specialist Centre. J Clin Med. 2016;5(8):66.
145 Thomeer M, Demedts M, Behr J, Buhl R Costabel U, Flower CD, et al. Multidisciplinary interobserver agreement in the diagnosis of idiopathic pulmonary fibrosis. Eur Respir J. 2008;31(3):585-91.

146 Jo HE, Glaspole IN, Levin KC, McCormack SR, Mahar AM, Cooper WA, et al. Clinical impact of the interstitial lung disease multidisciplinary service. Respirology. 2016; 21(8):1438-44.

147 Theegarten D, Müller HM, Bonella F, Wohlschlaeger J, Costabel U. Diagnostic approach to interstitial pneumonias in a single centre: report on 88 cases. Diagn Pathol. 2012; 7:160

148 White ES, Xia M, Murray S, Dyal R, Flaherty CM, Flaherty KR, et al. Plasma surfactant protein-D, matrix metalloproteinase- 7 , and osteopontin index distinguishes idiopathic pulmonary fibrosis from other idiopathic interstitial pneumonias. Am J Respir Crit Care Med. 2016;194(10):1242-51.

149 Morais A, Beltrão M, Sokhatska O, Costa D, Melo N, Mota P, et al. Serum metalloproteinases 1 and 7 in the diagnosis of idiopathic pulmonary fibrosis and other interstitial pneumonias. Respir Med. 2015;109(8): 1063-8.

150 Flaherty KR, King TE Jr, Raghu G, Lynch JP 3rd, Colby TV, Travis WD, et al. Idiopathic interstitial pneumonia: what is the effect of a multidisciplinary approach to diagnosis? Am J Respir Crit Care Med. 2004;170(8): 904-10.

151 Hutchinson JP, Fogarty AW, McKeever TM, Hubbard RB. In-hospital mortality after surgical lung biopsy for interstitial lung disease in the United States. 2000 to 2011. Am J Respir Crit Care Med. 2016;193(10):1161-7. 